\title{
FILLING THE GAP BETWEEN AMERICAN AND RUSSIAN OPTIONS: ADJUSTABLE REGRET
}

\author{
SAVAS DAYANIK AND MICHAEL LUDKOVSKI
}

\begin{abstract}
We study several infinite-horizon optimal multiple-stopping problems for (geometric) Brownian motion. In finance, they naturally span between the American and Russian option formulations in terms of price and reduced regret. In statistics, they are continuous-time examples of best-choice problems with multiple rights. We find explicit formulas for the value functions and describe completely optimal exercise strategies whenever one exists. We also conjecture a new characterization of the value function for the open problem of the Russian option for arithmetic Brownian motion with drift.
\end{abstract}

\section{INTRODUCTION}

Let $X$ be a (geometric) Brownian motion whose initial state is $x$, and $r \geq 0$ be a constant discount rate. We study the optimal multiple-stopping problem

$$
\sup _{\tau_{1}, \tau_{2}, \ldots, \tau_{n}} \mathbb{E}_{x}\left[e^{-r \tau_{n}}\left(\max _{1 \leq i \leq n} X_{\tau_{i}}\right)\right],
$$

where the supremum is taken over $n \geq 1$ stopping times $\tau_{1}, \ldots, \tau_{n}$ of the process $X$.

The value of (1) can be thought as the value of a perpetual financial option, which gives its holder $n$ rights to mark the price $X$ of a stock and pays her the (discounted) maximum of those $n$ recorded marks at the final exercise time. This closely resembles the Russian option

$$
\sup _{\tau} \mathbb{E}_{x}\left[e^{-r \tau}\left(\max _{0 \leq t \leq \tau} X_{t}\right)\right],
$$

where the supremum is taken over stopping times $\tau$ of the process $X$. The Russian option problem was introduced and solved by Shepp and Shiryaev [9, 10] for a geometric Brownian motion $X$ with drift $\mu$ and volatility $\sigma$; see also Duffie and Harrison [4] for the related arbitrage pricing problem.

L. Shepp and A. Shiryaev argued that, compared to a standard perpetual American option, the Russian option can reduce its holder's regret for not having stopped earlier. Indeed, unlike an American option which pays the holder the stock price at the exercise time, the

Date: 7 April 2006.

2000 Mathematics Subject Classification. Primary: 60G40; Secondary: 60J65, 62L15.

Key words and phrases. optimal multiple-stopping, Russian option, best-choice problems. 
Russian option pays the historical maximum of the stock price at the exercise time since the contract has been entered.

However, they also showed that in a Black-Scholes model this option's value equals infinity if the drift $\mu$ and discount rate $r$ are the same. In other words, if the stock follows a geometric Brownian motion and pays no dividend, then the price to be paid for the Russian option's "little or no regret" feature is unaffordable.

This raises the following interesting questions: is there an option that provides the holder a range of comfort/regret levels at affordable prices? Is there a family of options with various levels of regret that can be obtained by a potential holder for cheaper prices?

A multiple-stopping option as in (1) can provide an affirmative answer to both questions. Its value is always finite, and it is always cheaper than the Russian option. It reduces to a standard American option for $n=1$, and its value increases to that of the Russian option as the number of exercise rights $n$ increases to infinity. Hence, the family of multiple-stopping options spans the range between American and Russian options in terms of price and reduced regret.

Some of the above facts are immediate, and we establish others after solving the problem in (1). We give explicit formula for its value function and describe an explicit optimal multiple-stopping strategy whenever one exists.

We also solve the problem in (1) when $r=0$ and $X$ is a linear Brownian motion on the unit interval $[0,1]$ with absorbing boundary points. This is an example of best-choice problems with several rights to choose; see, e.g., Freeman [7] and Samuels [8]. In that context, the decision maker faces a trade-off between sparing some of her rights for future use, versus the possibility that $X$ is absorbed at the left boundary, after which the remaining rights are not useful any more. We give explicitly both the value function and an optimal multiple-stopping rule describing the best way of marking new records of $X$. We also solve the case where the terminal payoff is not " $\max _{1 \leq i \leq n} X_{\tau_{i}}$ " but " $\max _{1 \leq i \leq n}\left(K-X_{\tau_{i}}\right)^{+}$" for some constant $K \in[0,1]$, mimicking a Russian (perpetual lookback) put.

The remainder of this paper is organized as follows. Section 2 provides a rigorous formulation of our problem and contains the summary of the main results. Section 3 outlines the general method of solution. The proofs are given in Sections 4 and 5 . Finally, Section 6 is devoted to discussion about the structure of the Russian option (2) that can be gleaned from (1), including the open problem about the value of the Russian option for an arithmetic Brownian motion. 


\section{Problem Formulation AND main Results}

Let $X$ be a linear regular diffusion with state space $\mathcal{I} \subseteq \mathbb{R}$. Let $\mathcal{S} \equiv \mathcal{S}^{(1)}$ and

$$
\mathcal{S}^{(n)} \triangleq\left\{\left(\tau_{1}, \ldots, \tau_{n}\right): \text { each } \tau_{i} \text { is a stopping time of } X \text { and } \tau_{1} \leq \ldots \leq \tau_{n}\right\}, n \geq 1
$$

be the collection of all multiple-stopping rules for every fixed number of exercise rights $n$. Importantly, we allow strategies with multiple simultaneous exercises, $\tau_{k}=\tau_{k+1}$. Define the discounted optimal multiple-stopping problems

$$
V^{(n)}(x, m) \triangleq \sup _{\left(\tau_{1}, \ldots, \tau_{n}\right) \in \mathcal{S}(n)} \mathbb{E}_{x}\left[e^{-r \tau_{n}}\left(m \vee \max _{1 \leq i \leq n} h\left(X_{\tau_{i}}\right)\right)\right], \quad x, m \in \mathcal{I}, n \geq 1
$$

where $x \in \mathcal{I}$ is the initial value of the process $X, m \in \mathcal{I}$ is an initial lower cap on the terminal payoff, and $h(\cdot)$ is the payoff function. Note that the supremum in $(1)$ equals $V^{(n)}(x, 0)$ with $h(x)=x$.

A multiple-stopping rule $\left(\tau, \tau_{1}, \ldots, \tau_{n-1}\right) \in \mathcal{S}^{(n)}$ is optimal for $V^{(n)}$ if and only if (i) the rule $\left(\tau_{1}, \ldots, \tau_{n-1}\right)$ is optimal for $V^{(n-1)}$, and (ii) the optimal stopping rule $\tau$ maximizes the expected discounted future payoff $V^{(n-1)}\left(X_{\tau}, m \vee h\left(X_{\tau}\right)\right)$ obtained by following the optimal rule $\left(\tau_{1}, \ldots, \tau_{n-1}\right)$. This application of the dynamic programming principle can be made rigorous as in Carmona and Dayanik [1], Carmona and Touzi [2], and gives the relation

$$
V^{(n)}(x, m)=\sup _{\tau \in \mathcal{S}} \mathbb{E}_{x}\left[e^{-r \tau} V^{(n-1)}\left(X_{\tau}, m \vee h\left(X_{\tau}\right)\right)\right], \quad x, m \in \mathcal{I}, n \geq 1 .
$$

We set $V^{(0)}(x, m)=m$ for every $x, m \in \mathcal{I}$. Thus, the optimal multiple-stopping problem can be addressed by sequentially solving a family of optimal stopping problems.

2.1. Brownian motion. Let $r=0$ in (3), and $X_{t}=B_{t}$ be a Brownian motion on the interval $\mathcal{I}=[0,1]$ with absorbing endpoints.

Proposition 1. Let $h(x)=x$ for $x \in[0,1]$. Then, for every $m \in[0,1]$ and $n \geq 1$, we have the following:

(i) The value function $V^{(n)}(x, m)$ of $(3)$ is given by

$$
V^{(n)}(x, m)=\left\{\begin{array}{ll}
m+n\left(1-m^{1 / n}\right) x & x<m^{(n-1) / n} \\
n x-(n-1) x^{n /(n-1)} & x \geqslant m^{(n-1) / n}
\end{array}\right\}, \quad x \in[0,1] .
$$

(ii) The first exit time

$$
\tau_{m}^{(n)} \triangleq \inf \left\{t \geq 0: X_{t} \notin\left(0, m^{(n-1) / n}\right)\right\} \in \mathcal{S}
$$

of the process $X$ from the interval $\left(0, m^{(n-1) / n}\right)$ is optimal for the problem in (4). 


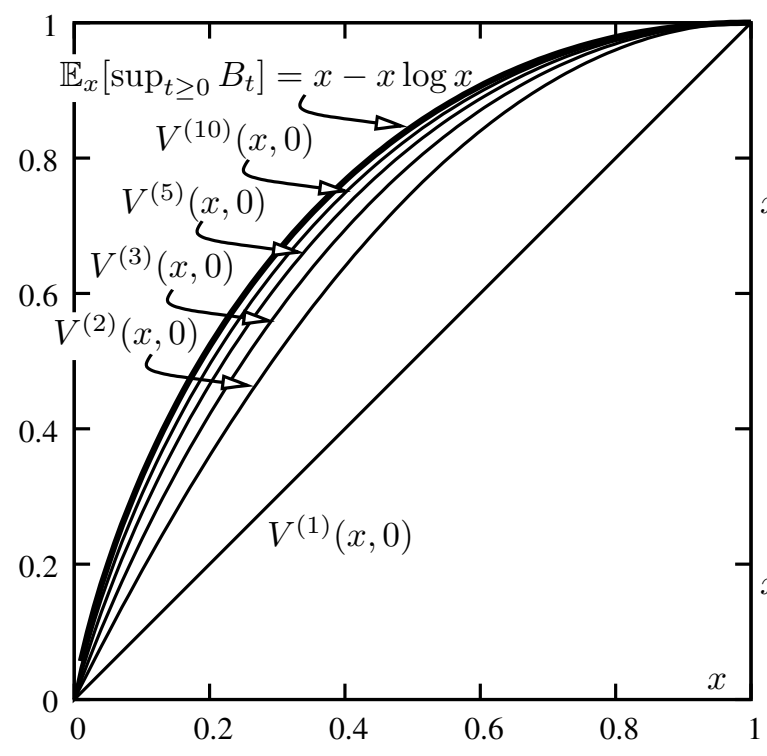

(a)

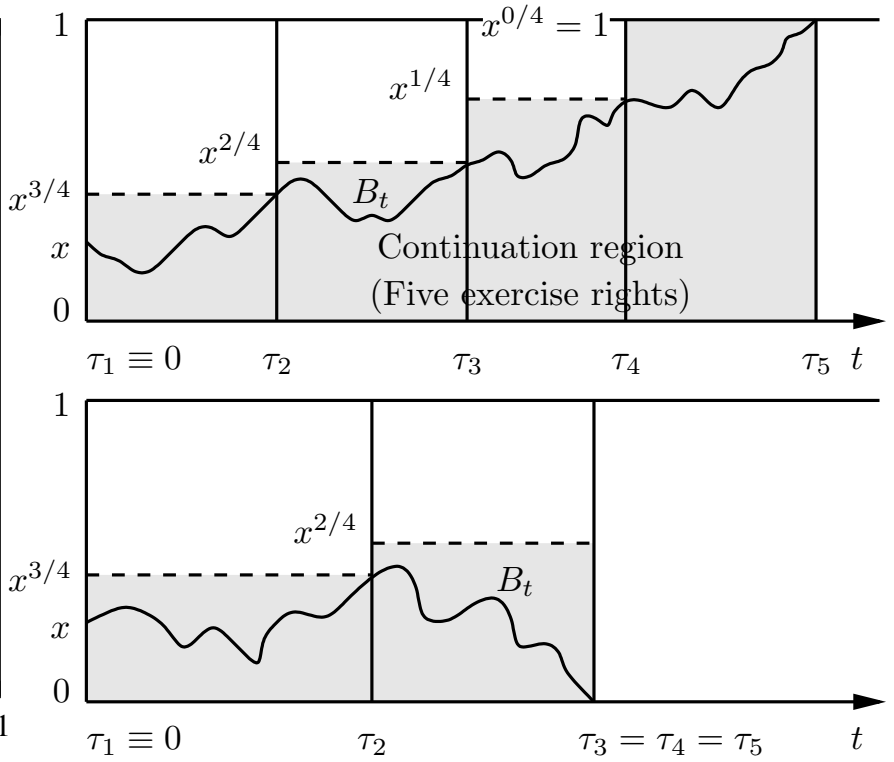

(b)

Figure 1. (a) Convergence of $V^{(n)}(x, 0)$ of (1) to $\mathbb{E}_{x}\left[\sup _{t \geq 0} B_{t}\right]$ for the linear Brownian motion on $\mathcal{I}=[0,1]$ with absorbing boundaries at endpoints. (b) Illustrations of optimal multiple-stopping rule $\left(\tau_{1}, \ldots, \tau_{5}\right)$ of Corollary 1 for two sample paths starting at $B_{0}=x$ when five exercise rights exist.

Corollary 1. If $h(x)=x$ for every $x \in[0,1]$, then an optimal multiple-stopping strategy $\left(\tau_{1}, \ldots, \tau_{n}\right) \in \mathcal{S}^{(n)}$ for the problem (1) is

$$
\tau_{1} \equiv 0, \quad \text { and } \quad \tau_{k+1}=\left.\tau_{m}^{(n-k)} \circ \theta_{\tau_{k}}\right|_{m=X_{\tau_{k}}}=\inf \left\{t \geq 0: X_{t} \notin\left(0, x^{(n-k-1) /(n-1)}\right)\right\}
$$

for every $k=1, \ldots, n-1$, where $\theta_{t}: \Omega \mapsto \Omega$ is the shift operator; i.e., $X_{s} \circ \theta_{t}=X_{t+s}$ for every $s, t \geq 0$.

Corollary 1 states that an optimal policy for the original problem (3) is as follows. Starting at any fixed $X_{0}=x$, we always make our first stop immediately. As long as the process $X$ is not absorbed at 0 , we exercise the remaining $n-1$ rights at each time that it reaches the increasing levels $x^{(n-k) /(n-1)}, k=2, \ldots, n$. Observe that the final $n$th right is always exercised when the process reaches the right boundary point $x^{(n-n) /(n-1)}=1$ if it has not been already absorbed at 0 . For example, starting at $x=1 / 4$ with five exercise rights, it is optimal to stop at the first passage times of $1 / 4=0.25,(1 / 4)^{3 / 4}=0.3536,(1 / 4)^{2 / 4}=0.5$, $(1 / 4)^{1 / 4}=0.707$ and $(1 / 4)^{0 / 4}=1$, as long as the process is not absorbed at 0 . In the latter case, the remaining rights are exercised immediately.

By (5) we have $V^{(n)}(x, 0)=n x-(n-1) x^{n /(n-1)}=x+(n-1)\left[1-x^{1 /(n-1)}\right] x$ for every $x \in[0,1]$ and $n \geq 1$. Therefore, $\lim _{n \rightarrow \infty} V^{(n)}(x, 0)=x-x \log x$. The next computation 
shows that this is $\mathbb{E}_{x}\left[\sup _{t \geq 0} B_{t}\right]$. Indeed, if we define $\tau_{a}=\inf \left\{s \geq 0 ; B_{s} \geq a\right\}$ for every $a \in(0,1]$, then the events $\left\{\sup _{t \geq 0} B_{t}>a\right\}$ and $\left\{\tau_{a}<\tau_{0}\right\}$ are the same, and

$$
\begin{aligned}
\mathbb{E}_{x}\left[\sup _{t \geq 0} B_{t}\right]=\int_{0}^{\infty} \mathbb{P}_{x}\left(\sup _{t \geq 0} B_{t}>a\right) d a & =\int_{0}^{x} \mathbb{P}_{x}\left(\tau_{a}<\tau_{0}\right) d a+\int_{x}^{1} \mathbb{P}_{x}\left(\tau_{a}<\tau_{0}\right) d a \\
& =x+\int_{x}^{1} \frac{x}{a} d a=x-x \log x .
\end{aligned}
$$

The left panel of Figure 1 shows graphically the convergence of the sequence $\left\{V^{(n)}(x, 0)\right\}_{n \geq 1}$ to the function $x \mapsto \mathbb{E}_{x}\left[\sup _{t \geq 0} B_{t}\right]=x(1-\log x)$ on $x \in[0,1]$.

Proposition 2. Let $0 \leq K \leq 1$, and $h(x)=(K-x)^{+}=\max (K-x, 0)$ for $x \in[0,1]$. For every $m \in[0, K]$ and $n \geq 1$, we have the following:

(i) The value function in (3) is given by

$$
V^{(n)}(x, m)=\left\{\begin{array}{ll}
K-1+n(1-x)-(n-1)(1-x)^{n /(n-1)}, & x<x_{m}^{(n)} \\
m+n(1-x)\left[1-(1+m-K)^{1 / n}\right], & x \geqslant x_{m}^{(n)}
\end{array}\right\},
$$

for every $x \in[0,1]$, where $x_{m}^{(n)}=1-(1+m-K)^{(n-1) / n}$.

(ii) The sequence $\left(x_{m}^{(n)}\right)_{n \geq 1}$ is decreasing and $x_{m}^{(n)}<K-m$.

(iii) The first exit time $\tau_{m}^{(n)} \triangleq \inf \left\{t \geq 0: X_{t} \notin\left(x_{m}^{(n)}, 1\right)\right\}$ of the process $X$ from the interval $\left(x_{m}^{(n)}, 1\right)$ is an optimal stopping time of (4).

For every $m \in(K, 1]$ and $n \geq 1$, we have $V^{(n)}(x, m)=m$ trivially.

Corollary 2. If $h(x)=(K-x)^{+}$for every $x \in[0,1]$, then an optimal multiple-stopping strategy $\left(\tau_{1}, \ldots, \tau_{n}\right) \in \mathcal{S}^{(n)}$ for the problem (1) is

$$
\begin{aligned}
\tau_{1} & =\inf \left\{t \geq 0: X_{t} \notin\left(1-(1-K)^{(n-1) / n}, 1\right)\right\}, \quad \text { and } \\
\tau_{k+1} & =\left.\tau_{m}^{(n-k)} \circ \theta_{\tau_{k}}\right|_{m=K-X_{\tau_{k}}}=\inf \left\{t \geq 0: X_{t} \notin\left(1-(1-K)^{(n-k-1) / n}, 1\right)\right\}
\end{aligned}
$$

for every $k=1, \ldots, n-1$.

2.2. Geometric Brownian motion. Suppose that $r>0$ and $h(x)=x, x \in \mathbb{R}_{+}$in $(3)$. Let

$$
X_{t}=x \exp \left\{\left(\mu-\frac{\sigma^{2}}{2}\right) t+\sigma B_{t}\right\}, \quad t \geq 0, x \in \mathcal{I}=[0, \infty)
$$

be a geometric Brownian motion with drift $\mu$ and volatility $\sigma$. Let us denote by $-k_{1}<0<$ $1 \leq k_{2}$ the roots of the quadratic equation $f(k) \triangleq\left(\sigma^{2} / 2\right) k^{2}+\left[\mu-\left(\sigma^{2} / 2\right)\right] k-r=0$; namely,

$$
-k_{1}, k_{2}=-\left(\frac{\mu}{\sigma^{2}}-\frac{1}{2}\right) \mp \sqrt{\left(\frac{\mu}{\sigma^{2}}-\frac{1}{2}\right)^{2}+\frac{2 r}{\sigma^{2}}} ; \quad \text { and } \quad k \triangleq k_{1}+k_{2} .
$$


FILLING THE GAP BETWEEN AMERICAN AND RUSSIAN OPTIONS: ADJUSTABLE REGRET

Proposition 3. Suppose that $\mu=r$. Define the increasing sequence

$$
a_{1}=1, \quad a_{n+1}=a_{n}+\frac{k_{1}^{k_{1}}}{k^{k}} a_{n}^{-k_{1}}, n=1,2, \ldots,
$$

Then, for every $n \geq 1$ and $m \in \mathbb{R}_{+}$, we have the following:

(i) The value function $V^{(n)}(x, m)$ in (3) is given by

$$
V^{(n)}(x, m)=\left\{\begin{array}{ll}
m, & 0 \leq x<x_{m}^{(n)} \triangleq \frac{k_{1}}{k} \cdot \frac{m}{a_{n}} \\
a_{n} x+\frac{k_{1}^{k_{1}} k^{k} a_{n}^{-k_{1}} m^{k} x^{-k_{1}},}{k^{2}} & x \geq x_{m}^{(n)}
\end{array}\right\}, \quad x \in \mathbb{R}_{+} .
$$

(ii) The sequence $\left(x_{m}^{(n)}\right)_{n \geq 1}$ is decreasing, and $x_{m}^{(n)}<m$.

(iii) For the problem in (4), immediate stopping is optimal if $m=0$, and no optimal stopping time exists if $m>0$, but for every $\varepsilon>0$, the first exit time

$$
\tau_{m}^{(n)}(\varepsilon) \triangleq \inf \left\{t \geq 0: X_{t} \notin\left(x_{m}^{(n)}, x_{m}^{(n)}(\varepsilon)\right)\right\}
$$

of the process $X$ from the open interval $\left(x_{m}^{(n)}, x_{m}^{(n)}(\varepsilon)\right)$ is $\varepsilon$-optimal, where

$$
x_{m}^{(n)}(\varepsilon)=\max \left\{m, \frac{k_{1}}{a_{n}}\left(\frac{m^{k}}{\varepsilon k^{k}}\right)^{1 / k_{1}}\right\} .
$$

Corollary 3. Suppose that $\mu=r$. The value of the multiple-stopping option in (1) with $n \geq 1$ exercise rights equals $V^{(n)}(x, 0)=a_{n} x$ at every initial stock price $x \in \mathbb{R}_{+}$. There is no optimal multiple-stopping strategy. However, for every $\varepsilon>0$, the strategy $\left(\tau_{1}(\varepsilon), \ldots, \tau_{n}(\varepsilon)\right) \in \mathcal{S}^{(n)}$ is e-optimal, if

$$
\tau_{1}(\varepsilon) \equiv 0, \quad \text { and } \quad \tau_{k+1}(\varepsilon)=\left.\tau_{m}^{(n-k)}(\varepsilon) \circ \theta_{\tau_{k}(\varepsilon)}\right|_{m=M_{k}(\varepsilon)}, \quad k=1, \ldots, n-1
$$

is the first exit time after $\tau_{k}(\varepsilon)$ of the process $X$ from the interval $\left(x_{m}^{(n-k)}, x_{m}^{(n-k)}(\varepsilon)\right)$ for $m=M_{k}(\varepsilon)$; here, $M_{k}(\varepsilon) \triangleq \max _{1 \leq i \leq k} X_{\tau_{i}(\varepsilon)}$ is the running maximum of $X_{\tau_{1}(\varepsilon)}, \ldots, X_{\tau_{n}(\varepsilon)}$.

Remark 1. Since the sequence $\left(a_{n}\right)_{n \geq 1}$ of $(12)$ is increasing, its limit as $n \rightarrow \infty$ exists and is greater than one. Taking limit as $n \rightarrow \infty$ of the recursion's both sides in (12) implies that $\lim _{n \rightarrow \infty} a_{n}=+\infty$. Therefore, for every $m \in \mathbb{R}_{+}$, we have $\lim _{n \rightarrow \infty} x_{m}^{(n)}=0$ and $\lim _{n \rightarrow \infty} V^{(n)}(x, m)=+\infty$, which is in agreement with the infinite value of the Russian option in the case that $\mu=r$; see Shepp and Shiryaev [10]. This behavior is illustrated in Figure 2. 

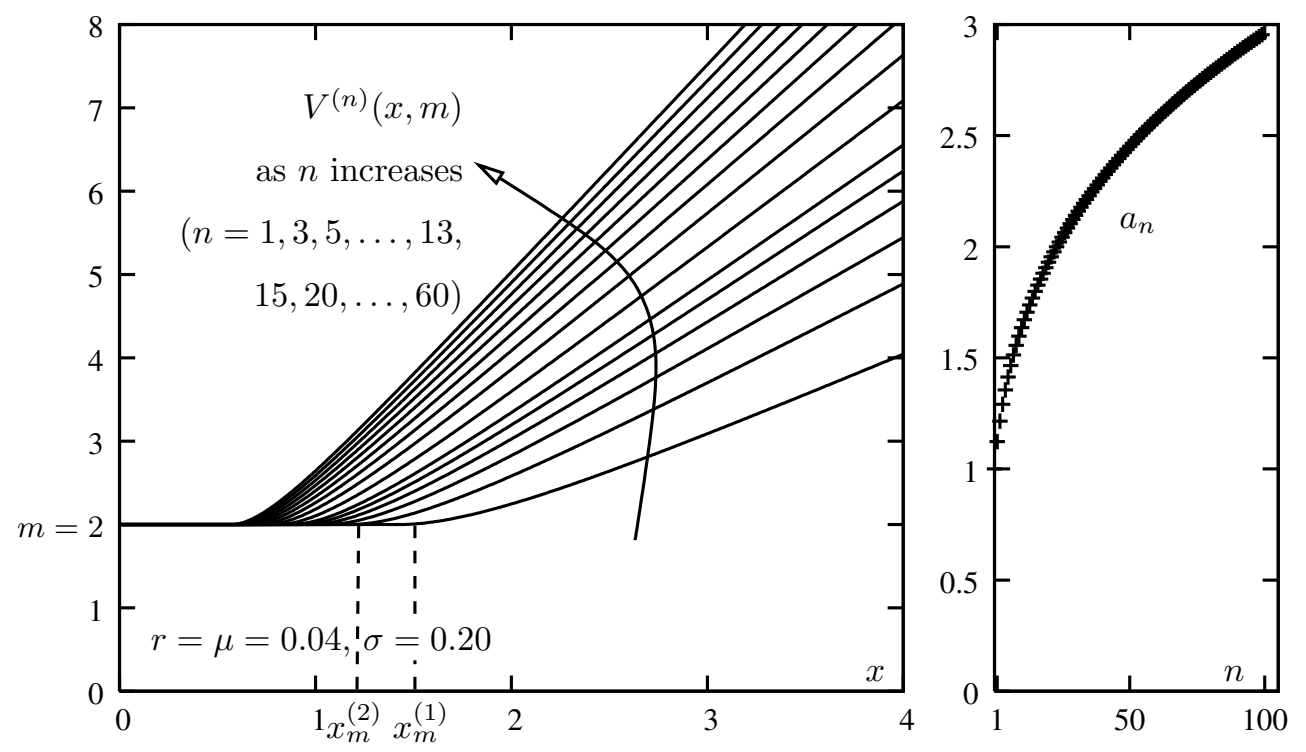

FiguRE 2. Behavior of $V^{(n)}(x, m)$ as a function of $n$. We take $\sigma=0.2, \mu=$ $r=0.04, m=2$. The left panel shows $V^{(n)}(x, m)$ for a range of $x$ around $m$ with $n=1,3,5, \ldots, 11,15,20, \ldots, 60$. The thresholds $x_{m}^{(n)}$ at which $V^{(n)}(x, m)$ "takes off" from the level $m$ are seen to be decreasing to zero as expected. The right panel shows the sequence $\left(a_{n}\right)$ from $(12)$. This sequence grows without limit; see Remark 1.

Proposition 4. Suppose that $\mu<r$. Define the increasing sequence

$$
\begin{aligned}
a_{1}=1, \quad a_{n+1} & =\frac{k_{1}}{k}\left[\left(\frac{k_{1}}{1+k_{1}}\right)^{1+k_{1}}\left(\frac{k_{2}}{k_{2}-1}\right)^{k_{2}-1}\right]^{-k_{2} / k} a_{n}^{k_{2}} \\
& +\frac{k_{2}}{k}\left[\left(\frac{k_{1}}{1+k_{1}}\right)^{1+k_{1}}\left(\frac{k_{2}}{k_{2}-1}\right)^{k_{2}-1}\right]^{k_{1} / k} a_{n}^{-k_{1}}, \quad n=1,2, \ldots
\end{aligned}
$$

Then, for every $n \geq 1$ and $m \in \mathbb{R}_{+}$, we have the following:

(i) The value function $V^{(n)}(x, m)$ in (3) is given by

$$
V^{(n)}(x, m)=\left\{\begin{array}{ll}
m, & 0 \leq x<x_{m, 1}^{(n)} \\
\ell_{m}^{(n)} \triangleq \frac{m}{k}\left[k_{1}\left(\frac{x}{x_{m, 1}^{(n)}}\right)^{k_{2}}+k_{2}\left(\frac{x}{x_{m, 1}^{(n)}}\right)^{-k_{1}}\right], & x_{m, 1}^{(n)} \leq x<x_{m, 2}^{(n)} \\
a_{n} x, & x \geq x_{m, 2}^{(n)}
\end{array}\right\}, \quad x \in \mathbb{R}_{+},
$$


where

$$
\begin{aligned}
& x_{m, 1}^{(n)} \triangleq\left(\frac{k_{1}}{1+k_{1}}\right)^{\left(1+k_{1}\right) / k}\left(\frac{k_{2}}{k_{2}-1}\right)^{\left(k_{2}-1\right) / k} \frac{m}{a_{n}} \\
& x_{m, 2}^{(n)} \triangleq\left(\frac{k_{1}}{1+k_{1}}\right)^{k_{1} / k}\left(\frac{k_{2}}{k_{2}-1}\right)^{k_{2} / k} \frac{m}{a_{n}}=\left[\frac{\left(1+k_{1}\right) k_{2}}{k_{1}\left(k_{2}-1\right)}\right]^{1 / k} x_{m, 1}^{(n)} .
\end{aligned}
$$

(ii) The sequences $\left(x_{m, 1}^{(n)}\right)_{n \geq 1}$ and $\left(x_{m, 2}^{(n)}\right)_{n \geq 1}$ are decreasing, and $x_{m, 1}^{(n)}<m<x_{m, 2}^{(n)}$.

(iii) The first exit time

$$
\tau_{m}^{(n)} \triangleq \inf \left\{t \geq 0: X_{t} \notin\left(x_{m, 1}^{(n)}, x_{m, 2}^{(n)}\right)\right\}
$$

of the process $X$ from the interval $\left(x_{m, 1}^{(n)}, x_{m, 2}^{(n)}\right)$ is an optimal stopping time for the problem in (4).

Remark 2. If $\mu=r$, then $k_{2}=1$ and $k=1+k_{1}$. As $\mu \nearrow r$, we have $k_{2} \searrow 1$, and the sequence $\left(a_{n}\right)_{n \geq 1}$ in (14) reduces to that in (12). Therefore, the $\left(a_{n}\right)$ in (12) and (14) are the same sequence, whose form is determined implicitly by the relation between $r$ and $\mu$.

Corollary 4. Suppose that $\mu<r$. The value of the multiple-stopping option in (1) with $n \geq 1$ exercise rights equals $V^{(n)}(x, 0)=a_{n} x$ at every initial stock price $x \in \mathbb{R}_{+}$. If

$$
\tau_{1} \equiv 0, \quad \text { and } \quad \tau_{k+1}=\left.\tau_{m}^{(n-k)} \circ \theta_{\tau_{k}}\right|_{m=M_{k}}, \quad k=1, \ldots, n-1
$$

is the first exit time after $\tau_{k}$ of the process $X$ from the interval $\left(x_{m, 1}^{(n-k)}, x_{m, 2}^{(n-k)}\right)$ for $m=M_{k} \triangleq$ $\max _{1 \leq i \leq k} X_{\tau_{i}}$, then the strategy $\left(\tau_{1}, \ldots, \tau_{n}\right) \in \mathcal{S}^{(n)}$ is optimal for the problem in (3).

Figure 3 illustrates the implementation of the optimal policy with $n \geq 5$ exercise rights along a sample path of the process $X$. One can make the following observations:

(i) Between successive exercises, the continuation region is a bounded interval containing the running maximum of $\left\{X_{\tau_{1}}, \ldots, X_{\tau_{n}}\right\}$.

(ii) Waiting time between two exercises is positive if and only if the process $X$ leaves the current continuation region from its upper boundary. Note that $\tau_{1}<\tau_{2}<\tau_{3}=\tau_{4}=$ $\ldots=\tau_{n}$ in Figure 3. As soon as the process leaves one of the continuation regions from its lower boundary, all of the remaining rights are exercised instantaneously.

Hence, left-boundaries of continuation regions provide protection again deteriorating time-value of the option, while right-boundaries enhance the terminal payoff by marking new records of the process.

(iii) Recall that an optimal exercise rule for the Russian option in (2) is the first time $\tau_{R}$ that the process $X$ reaches to the $(1 / \alpha)$ th-fraction of its running maximum $M(t) \triangleq$ 


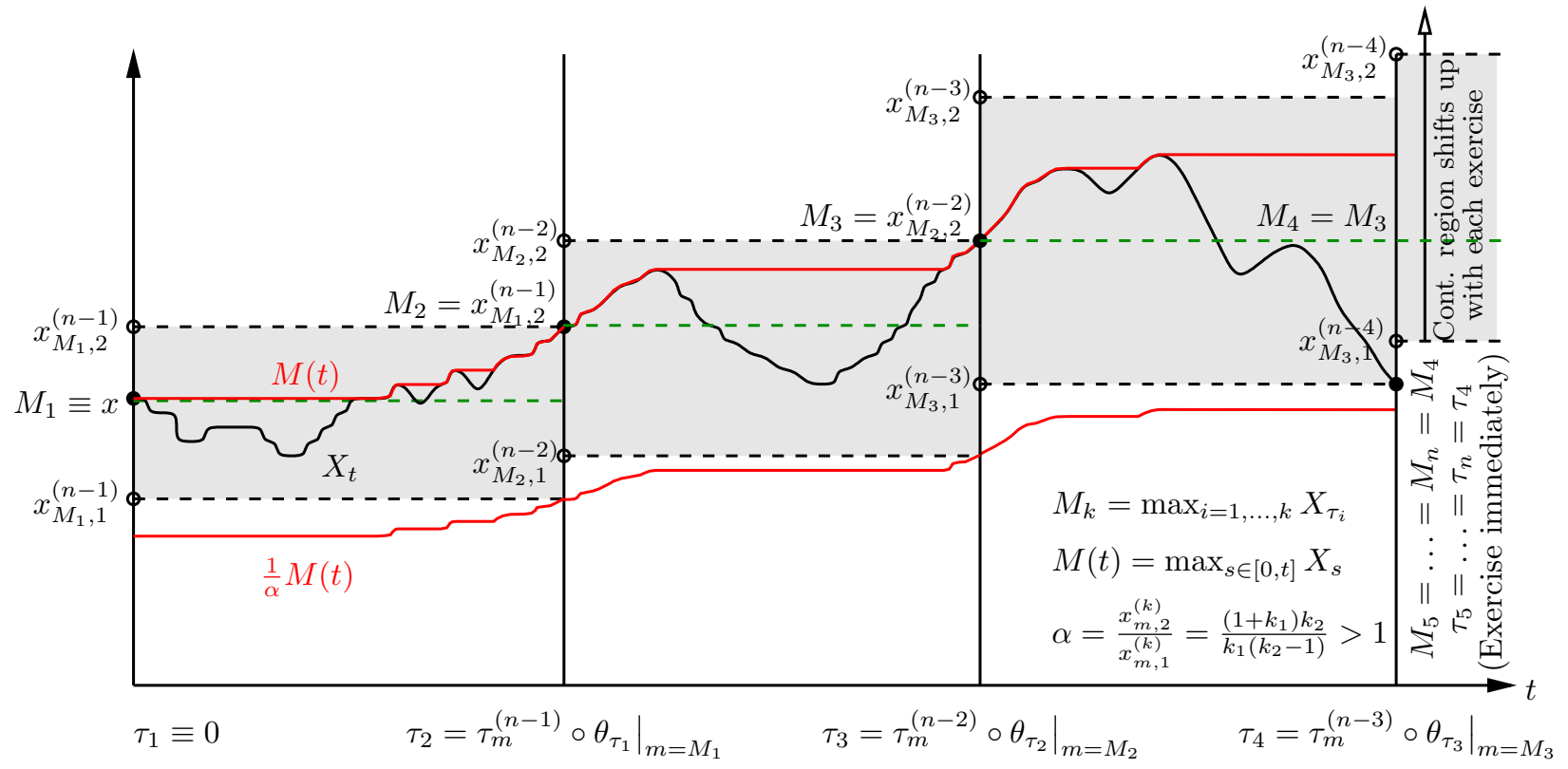

Figure 3. Geometric Brownian motion with $\mu<r$. Execution of the optimal policy when $n \geq 5$ along a sample path.

$\max _{s \in[0, t]} X_{t}$; see, e.g., Shepp and Shiryaev [10]:

$$
\tau_{R} \triangleq \inf \left\{t \geq 0: X_{t} \leq \frac{M(t)}{\alpha}\right\}, \quad \text { where } \quad \alpha \triangleq \frac{\left(1+k_{1}\right) k_{2}}{k_{1}\left(k_{2}-1\right)}>1
$$

The number $\alpha$ is also the ratio of upper and lower boundaries $x_{m, 2}^{(n)}$ and $x_{m, 1}^{(n)}$ in 16 . of continuation region of the optimal multiple-stopping problem for every $n \geq 1$ and $m \in \mathbb{R}_{+}$.

In Figure 3, the lower boundaries $x_{M_{j}, 1}^{(n-j)}, j=1, \ldots, n$ of continuation regions always lay above the exercise boundary $t \mapsto M(t) / \alpha$ of the Russian option. In other words, the continuation region of the multiple optimal-stopping is contained in that of the Russian option. If $\left(\tau_{1}, \ldots, \tau_{n}\right)$ is the multiple optimal-stopping strategy described in Corollary 4, then it is easy to show that $\tau_{1} \leq \cdots \leq \tau_{n} \leq \tau_{R}$.

Proposition 5. Suppose that $\mu<r$. Then, for every $m \in \mathbb{R}_{+}$, we have

$$
\begin{gathered}
a \triangleq \lim _{n \rightarrow \infty} a_{n}=\left(\frac{k_{1}}{1+k_{1}}\right)^{k_{1} / k}\left(\frac{k_{2}}{k_{2}-1}\right)^{k_{2} / k}, \\
x_{m, 1} \triangleq \lim _{n \rightarrow \infty} x_{m, 1}^{(n)}=\left[\frac{k_{1}\left(k_{2}-1\right)}{\left(1+k_{1}\right) k_{2}}\right]^{1 / k} m<m=\lim _{n \rightarrow \infty} x_{m, 2}^{(n)},
\end{gathered}
$$



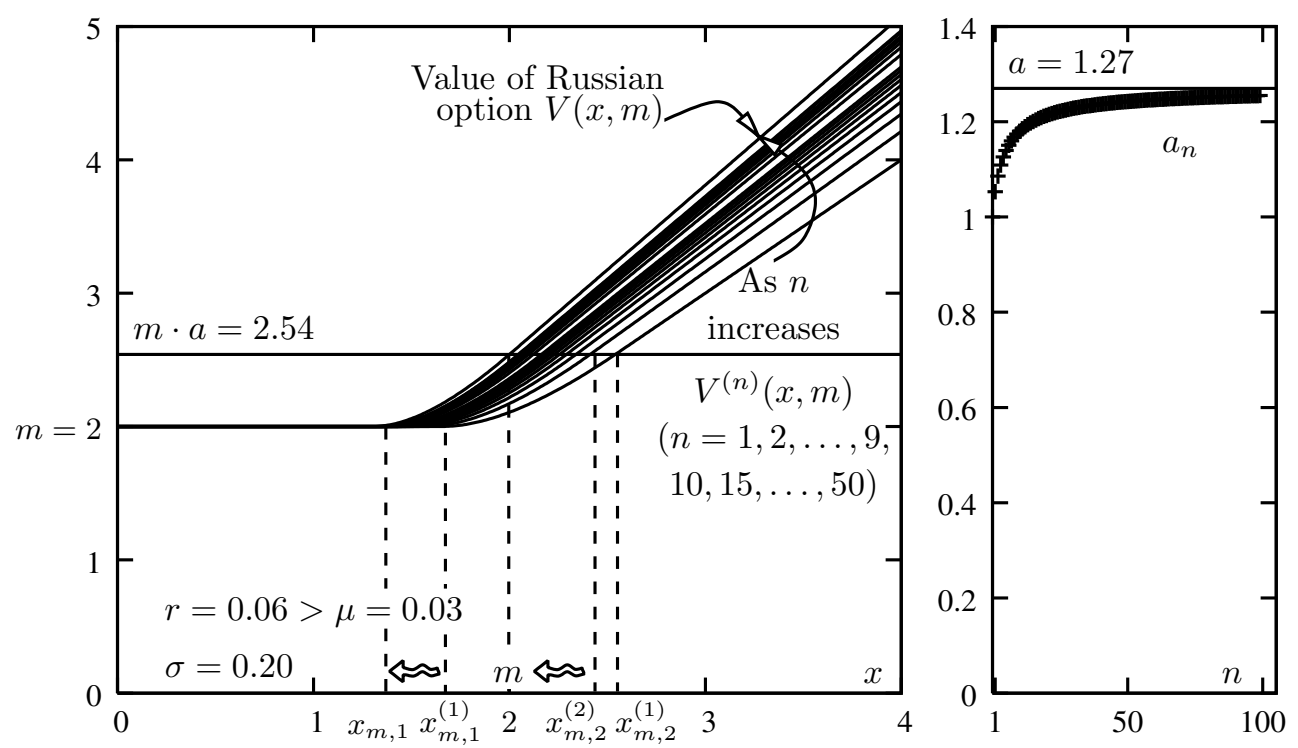

Figure 4. Behavior of $V^{(n)}(x, m)$ as a function of $n$ in Proposition 4 . We take $\sigma=0.2, \mu=0.03, r=0.06, m=2$. The left panel shows $V^{(n)}(x, m)$ for a range of $x$ around $m$ with $n=1,2, \ldots, 10,15,20, \ldots, 50$. The thresholds $x_{m, 1}^{(n)}$ are the "take-off" points at which $V^{(n)}(x, m)$ becomes larger than $m$ and are seen to be decreasing and approaching the limit $x_{m, 1}$ of Proposition 5. The horizontal line at $m \cdot a$ and each $V^{(n)}(x, m)$ intersect at the threshold $x=x_{m, 2}^{(n)}$ by Proposition 4. The mapping $x \mapsto V^{(n)}(x, m)=a_{n} x$ is linear on $\left[x_{m, 2}^{(n)}, \infty\right)$. The right panel shows the sequence $\left(a_{n}\right)_{n \geq 1}$ from (14). This sequence converges to $a$ of Proposition 5 and indicated with the solid line.

and the limit $V(x, m) \triangleq \lim _{n \rightarrow \infty} V^{(n)}(x, m)$ exists and equals

$$
V(x, m)=\left\{\begin{array}{ll}
m, & 0 \leq x<x_{m, 1} \\
\frac{m}{k}\left[k_{1}\left(\frac{x}{x_{m, 1}}\right)^{k_{2}}+k_{2}\left(\frac{x}{x_{m, 1}}\right)^{-k_{1}}\right], & x_{m, 1} \leq x<m \\
a x, & x \geq m
\end{array}\right\}, \quad x \in \mathbb{R}_{+} .
$$

Remark 3. For every $s \in \mathbb{R}_{+}$, the function $V(x, s), x \in[0, s]$ in (17) coincides with the value function of the Russian option calculated by Shepp and Shiryaev [10, Equation 2.4] (our $-k_{1}, k_{2}, x_{m, 1} / m$ are their $\gamma_{1}, \gamma_{2}, \alpha$, respectively).

Moreover, the identity $V(x, 0)=V(x, x), x \in \mathbb{R}_{+}$shows that, if the initial stock price is $x$, then the limiting value function $\lim _{n \rightarrow \infty} V^{(n)}(x, 0)=V(x, 0)$ of multiple-stopping option agrees with the Russian option's value function $V(x, x)$. Hence, as the number of exercise 
rights increases the regret of the holder for buying finite number of exercise rights instead of a full lookback option reduces to zero.

Finally, since the upper exercise boundary $x_{m, 2}^{(n)}$ of the optimal multiple-stopping problem converges as $n \rightarrow \infty$ to the "running maximum" $m$, the Russian option may be thought loosely as a multiple-stopping option with unlimited number of exercise rights, which are used to mark every time the underlying process breaks a record. See Figure 4 for an illustration of the convergence of the sequences $\left\{V^{(n)}(x, m)\right\}_{n \geq 1},\left(x_{m, 1}^{(n)}\right)_{n \geq 1},\left(x_{m, 2}^{(n)}\right)_{n \geq 1}$, and $\left(a_{n}\right)_{n \geq 1}$.

\section{Method OF SOLUtion}

The relation (4) lets us calculate the functions $V^{(n)}, n=1,2, \ldots$ recursively. After $V^{(n-1)}$ is calculated for some $n=1,2, \ldots$, let

$$
g_{m}^{(n)}(x) \triangleq V^{(n-1)}(x, h(x) \vee m), \quad x, m \in \mathcal{I} .
$$

Then (4) becomes a discounted optimal stopping problem with terminal payoff function $g_{m}^{(n)}$. In order to solve it, let us introduce the functions

$$
\begin{gathered}
\psi(x)=\left\{\begin{array}{ll}
\mathbb{E}_{x}\left[\exp \left\{-r \tau_{c}\right\}\right], & x<c \\
1 / \mathbb{E}_{c}\left[\exp \left\{-r \tau_{x}\right\}\right], & x \geq c
\end{array}\right\}, \quad \varphi(x)=\left\{\begin{array}{ll}
1 / \mathbb{E}_{c}\left[\exp \left\{-r \tau_{x}\right\}\right], & x<c \\
\mathbb{E}_{x}\left[\exp \left\{-r \tau_{c}\right\}\right], & x \geq c
\end{array}\right\}, \quad \text { and } \\
F(x)=\frac{\psi(x)}{\varphi(x)}, \quad x \in \mathcal{I},
\end{gathered}
$$

where $c$ is an arbitrary but fixed point in the interior of the state space $\mathcal{I}$, and the random variable $\tau_{y}$ is the first passage time of $X$ to the level $y \in \mathcal{I}$. The functions $\psi(\cdot)$ and $\varphi(\cdot)$ are increasing and decreasing, respectively, and are the only (up to multiplication by positive constants) monotonic solutions of the differential equation

$$
\mathcal{A} u(x)-r u(x)=0 .
$$

Here $\mathcal{A}$ is the infinitesimal generator of the process $X$ and coincides on smooth functions with the differential operator

$$
\mathcal{A} u(x)=\frac{\sigma^{2}}{2} u^{\prime \prime}(x)+\mu u^{\prime}(x) \quad \text { and } \quad \mathcal{A} u(x)=\frac{\sigma^{2}}{2} x^{2} u^{\prime \prime}(x)+\mu x u^{\prime}(x)
$$

for arithmetic (i.e., $X_{t}=X_{0}+\mu t+\sigma B_{t}, t \geq 0$ ) and geometric Brownian motion, respectively. Both of these processes are linear regular diffusions, and the boundaries, denoted by $a<b$, of their state-spaces are natural. Therefore, the results of Dayanik and Karatzas [3, Subsection 5.2] apply, and we summarize here their direct implications for the problem in (4): 
Proposition 6. (i) The function $x \mapsto V^{(n)}(x, m)$ is either identically infinite or finite everywhere. It is finite if and only if the limits

$$
\limsup _{x \downarrow a} \frac{g_{m}^{(n)} \vee 0}{\varphi} \circ F^{-1}(x) \quad \text { and } \quad \limsup _{x \uparrow b} \frac{g_{m}^{(n)} \vee 0}{\psi} \circ F^{-1}(x) \text { are finite. }
$$

(ii) If the function $x \mapsto V^{(n)}(x, m)$ is finite, then $V^{(n)}(x, m)=\varphi(x) \cdot W_{m}^{(n)}(F(x))$ for every $x, m \in \mathcal{I}$, where $W_{m}^{(n)}$ is the smallest nonnegative concave majorant of the function

$$
G_{m}^{(n)}(y) \triangleq\left(\frac{g_{m}^{(n)}}{\varphi}\right) \circ F^{-1}(y), \quad y \in F(\mathcal{I})
$$

(iii) If an optimal stopping time exists for (4), then the first exit time of the process $X$ from the continuation region $C_{m}=\left\{x \in \mathcal{I}: V^{(n)}(x, m)>g_{m}^{(n)}(x)\right\}$ is also optimal.

(iv) An optimal stopping time exists if the limits in part (i) are zero. If one of the limits is positive, then an optimal stopping time exists if and only if the associated boundary point is a limit point of the stopping region $\mathcal{I} \backslash C_{m}$.

\section{Brownian motion without discounting: proofs of Propositions 1 and 2}

We begin by analyzing the problems stated in Section 2.1. In this case because $X$ is a linear Brownian motion and there is no discounting, we can use the classical tool of concave majorization as in the original paper of Dynkin [5]; see also Dynkin and Yushkevich [6]. No transformation is necessary allowing for a more intuitive proof based on direct geometric reasoning.

Fix $m \in[0,1]$ and let $g_{m}^{(n)}(x)=V^{(n-1)}(x, h(x) \vee m)$. Since $V^{(n)}(x, m)=\sup _{\tau \in \mathcal{S}} \mathbb{E}_{x}\left[g_{m}^{(n)}\left(X_{\tau}\right)\right]$ it follows that $x \mapsto V^{(n)}(x, m)$ is the smallest concave majorant on $[0,1]$ of $g_{m}^{(n)}(x)$.

To give the reader some intuition, let us carry out explicitly the first couple of steps that lead to Proposition 1. Let us look at the case that $h(x)=x$ for every $x \in[0,1]$. First, if no stopping times are left, then trivially $V^{(0)}(x, m)=m$. Hence $g_{m}^{(1)}(x)=x \vee m$ and it is easy to see that the concave majorant of $g_{m}^{(1)}(x)$ is the affine function

$$
V^{(1)}(x, m)=m+(1-m) x .
$$

Also since $V^{(1)}(x, m)>g_{m}^{(1)}(x)$ everywhere except at the boundaries, the stopping region is $\{0,1\}$. This shows that given one exercise right, an optimal strategy is to wait until hitting the absorbing boundary points. Next,

$$
g_{m}^{(2)}(x)=V^{(1)}(x, x \vee m)= \begin{cases}m+(1-m) x, & x<m, \\ x+(1-x) x, & x \geqslant m .\end{cases}
$$

Hence, $g^{(2)}(x)$ is linear for small $x$, and then concave quadratic for large $x$. Note that $\left.(d / d x) g_{m}^{(2)}(x)\right|_{x=m-}=1-m$, while $\left.(d / d x) g_{m}^{(2)}(x)\right|_{x=m+}=2(1-m)>1-m$. Therefore, we 


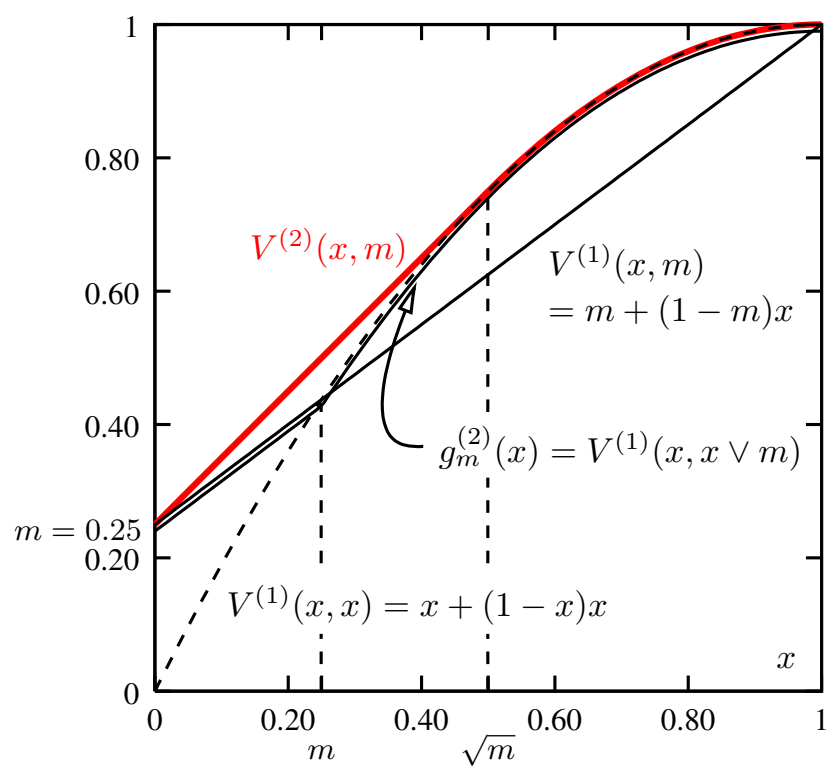

Figure 5. Plot of $V^{(1)}(x, 1 / 4), g_{1 / 4}^{(2)}(x)$ and $V^{(2)}(x, 1 / 4)$ for the linear Brownian motion on $[0,1]$.

have a "corner" at $x=m$. As Figure 5 illustrates, the smallest concave majorant of $g_{m}^{(2)}(x)$ is given by

$$
V^{(2)}(x, m)= \begin{cases}m+2(1-\sqrt{m}) x, & x<\sqrt{m}, \\ 2 x-x^{2}, & x \geqslant \sqrt{m} .\end{cases}
$$

Moreover, we see that the continuation region is $\left(0, x_{m}^{(2)}\right)$ where the threshold $x_{m}^{(2)}=\sqrt{m}$ solves the slope-matching equation $\left(g_{m}^{(2)}(x)-m\right) / x=(d / d x) g_{m}^{(2)}(x)$.

Proceeding in this way one obtains the result of Proposition 1 by induction. We have already confirmed (5) for $n=1,2$. Assuming (5) is true for $n$, then

$$
g_{m}^{(n+1)}(x)=V^{(n)}(x, x \vee m)= \begin{cases}m+n\left(1-m^{1 / n}\right) x, & x<m, \\ (n+1) x-n x^{(n+1) / n}, & x \geqslant m .\end{cases}
$$

The smallest concave majorant of $g_{m}^{(n+1)}(x)$ is linear on $\left[0, x_{m}^{(n+1)}\right]$ and is $g_{m}^{(n+1)}(x)$ itself on $\left[x_{m}^{(n+1)}, 1\right]$. At the critical threshold $x_{m}^{(n+1)}$ the slopes of the two pieces match, yielding

$$
(n+1)-\left.(n+1) x^{1 / n}\right|_{x=x_{m}^{(n+1)}}=\left.\frac{(n+1) x-n x^{(n+1) / n}-m}{x}\right|_{x=x_{m}^{(n+1)}}
$$

or $x_{m}^{(n+1)}=m^{1 /(n+1)}$ as claimed. The continuation region is connected because $g_{m}^{(n+1)}(x)$ is concave on $\left[x_{m}^{(n+1)}, 1\right] \subset[m, 1]$. This recovers (5) for $n$ replaced with $n+1$, completing the induction step. 
The proof of Proposition 2 is very similar, except we must take $h(x)=(K-x)^{+}$in (3). To start the induction, we compute

$$
V^{(1)}(x, m)=K-(K-m) x
$$

and $V^{(2)}(x, m)$ is the smallest concave majorant of $V^{(1)}\left(x,(K-x)^{+} \vee m\right)$; i.e.,

$$
V^{(2)}(x, m)= \begin{cases}K-x^{2}, & x<1-\sqrt{1+m-K} \\ m+2(1-x)(1-\sqrt{1+m-K}), & x \geq 1-\sqrt{1+m-K}\end{cases}
$$

Proposition 2 now easily follows by induction. Observe that $V^{(n)}(x, m)$ is linear on $\left[x_{m}^{(n)}, 1\right]$ and concave on $\left[0, x_{m}^{(n)}\right]$. The limit can be obtained with a computation similar to (8) yielding

$$
\mathbb{E}_{x}\left[\sup _{\tau}\left(K-B_{\tau}\right)^{+}\right]= \begin{cases}(x-1) \log (1-K), & x \geq K \\ (K-x)+(x-1) \log (1-x), & x<K .\end{cases}
$$

5. Geometric Brownian motion with discounting: proofs of Propositions 3 , 4. AND 5

5.1. Proof of Proposition 3. We shall start by proving (i). By an induction on $n$, we will establish simultaneously the identities 13 and

$$
W_{m}^{(n)}(y)= \begin{cases}m y^{k_{1} / k}, & 0 \leq y \leq y_{m}^{(n)} \triangleq\left(\frac{k_{1}}{k} \cdot \frac{m}{a_{n}}\right)^{k}<m^{k}, \\ L_{m}^{(n)}(y) \triangleq a_{n} y+\frac{k_{1}^{k_{1}}}{k^{k}} a_{n}^{-k_{1}} m^{k}, & y>y_{m}^{(n)} .\end{cases}
$$

For $n=1$, we have $g_{m}^{(1)}(x)=x \vee m$, and

$$
\limsup _{x \downarrow 0} \frac{g_{m}^{(1)}(x)}{\varphi(x)}=\lim _{x \downarrow 0} \frac{m}{x^{-k_{1}}}=0, \quad \limsup _{x \uparrow \infty} \frac{g_{m}^{(1)}(x)}{\psi(x)}=\lim _{x \uparrow \infty} \frac{x}{x}=1 .
$$

By Proposition 6(i), the function $V^{(1)}(\cdot, m)$ is finite. The smallest nonnegative concave majorant $W_{m}^{(1)}(y)$ of the function

$$
G_{m}^{(1)}(y)=\frac{g_{m}^{(1)}}{\varphi} \circ F^{-1}(y)= \begin{cases}m y^{k_{1} / k}, & 0 \leq y \leq m^{k} \\ y, & y>m^{k}\end{cases}
$$

coincides on $\left[0, y_{m}^{(1)}\right]$ with $y m^{k_{1} / k}$, and on $\left[y_{m}^{(1)}, \infty\right)$ with the affine function $L_{m}^{(1)}(y)$ which has slope one and is tangent to $y \mapsto m y^{k_{1} / k}$ at $y=y_{m}^{(1)}$; see Figure 6(a,b). The equations

$$
1=\left.\frac{d}{d y}\left(m y^{k_{1} / k}\right)\right|_{y=y_{m}^{(1)}}, \quad L_{m}^{(1)}\left(y_{m}^{(1)}\right)=\left.m y^{k_{1} / k}\right|_{y=y_{m}^{(1)}}
$$




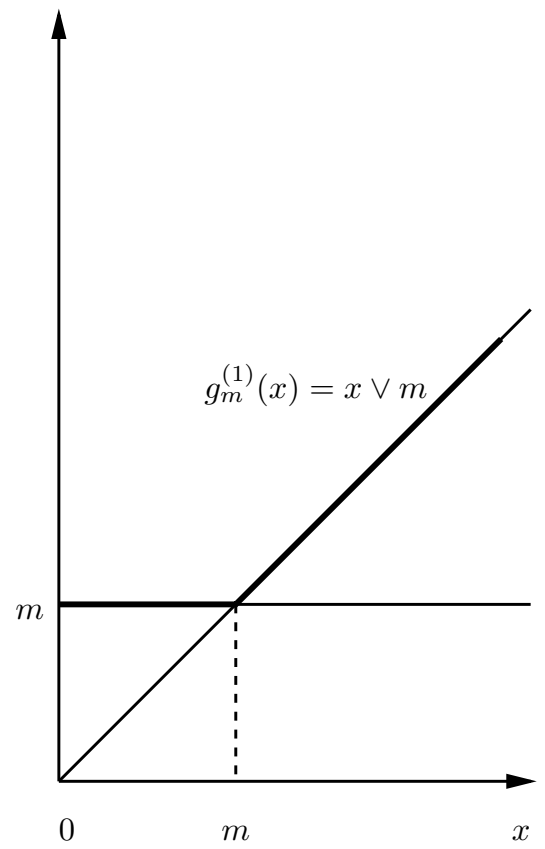

(a)

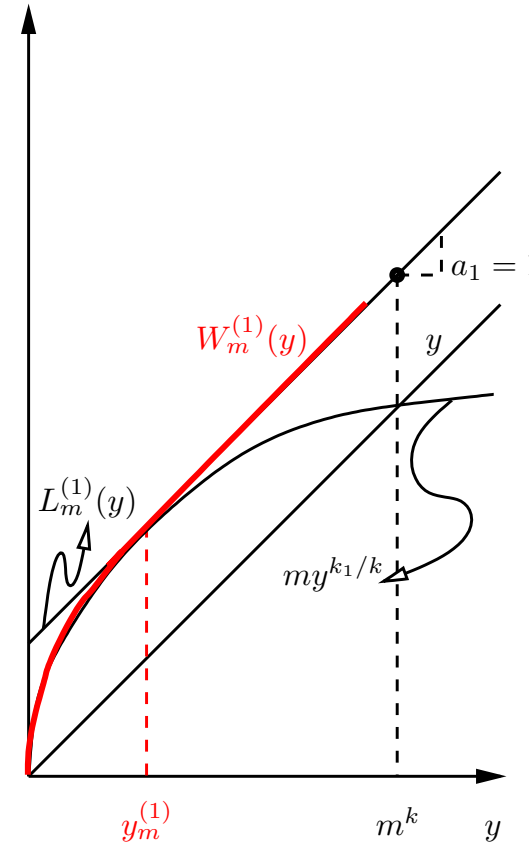

(b)

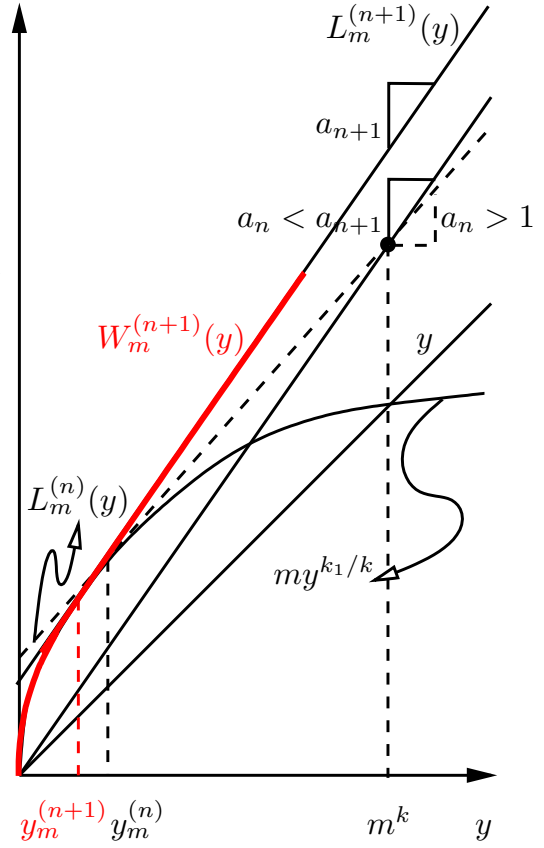

(c)

Figure 6. Illustrations for the proof of Proposition 3. In (b) and (c), $n \geq 1$, and $L_{m}^{(n)}(y)$ is the straight line, which is parallel to $y \mapsto a_{n} y$ and tangent to $y \mapsto m y^{k_{n} / k}$ at $y=y_{m}^{(n)}$. This line intersects with $y \mapsto a_{n+1} y$ at $y=m^{k}$. In (b), $W_{m}^{(1)}(y)$ is the smallest nonnegative concave majorant of $G_{m}^{(1)}(y)$, which coincides on $\left[0, m^{k}\right]$ with $m y^{k_{1} / k}$ and on $\left[m^{k}, \infty\right)$ with $y$. In $(\mathrm{c}), W_{m}^{(n+1)}(y)$ is the same majorant of $G_{m}^{(n+1)}(y)$, which coincides on $\left[0, y_{m}^{(n)}\right]$ with $m y^{k_{1} / k}$, on $\left[y_{m}^{(n)}, m^{k}\right]$ with $L_{m}^{(n)}(y)$, and on $\left[m^{k}, \infty\right)$ with $a_{n+1} y$.

imply that

$$
W_{m}^{(1)}(y)= \begin{cases}m y^{k_{1} / k}, & 0 \leq y<y_{m}^{(1)} \equiv\left(\frac{k_{1}}{k} \cdot m\right)^{k}<m^{k}, \\ L_{m}^{(1)}(y)=y+\frac{k_{1}^{k_{1}}}{k^{k}} m^{k}, & y \geq y_{m}^{(1)} .\end{cases}
$$

Finally, if we define $x^{(1)}=F^{-1}\left(y_{m}^{(1)}\right)=\left(k_{1} / k\right) m<m$ and $\ell_{m}^{(1)}(x)=\varphi(x) L_{m}^{(1)}(F(x))$, then Proposition 6 (ii) and (iv) imply that

$$
V^{(1)}(x, m)=\varphi(x) W_{m}^{(1)}(F(x))= \begin{cases}m, & 0 \leq x<x_{m}^{(1)}=\frac{k_{1}}{k} m<m, \\ \ell_{m}^{(1)}(x)=x+\frac{k_{1}^{k_{1}}}{k^{k}} m^{k} x^{-k_{1}}, & x \geq x_{m}^{(1)} .\end{cases}
$$


Hence, both (13) and (19) hold for $n=1$ since $a_{1}=1$ by definition. Note also that, since the second limit in Proposition 6(i) is positive, and the right boundary $+\infty$ is not a limit point of the "stopping region" $\left\{x \in \mathbb{R}_{+}: V^{(1)}(x, m)=g_{m}^{(1)}(x)\right\}$ unless $m=0$, there is no optimal stopping time by Proposition 6(iv) if and only if $m>0$.

Suppose now that 13 and $(19)$ hold for some $n \geq 1$. Let us show that they are also correct for $n+1$. Recall that

$$
V^{(n+1)}(x, m)=\sup _{\tau \in \mathcal{S}} \mathbb{E}_{x}\left[e^{-r \tau} g_{m}^{(n+1)}\left(X_{\tau}\right)\right], \quad x, m \in \mathbb{R}_{+},
$$

where $g_{m}^{(n+1)}(x) \triangleq V^{(n)}(x, m \vee x)$ equals

$$
g_{m}^{(n+1)}(x)=\left\{\begin{array}{ll}
V^{(n)}(x, m), & 0 \leq x<m \\
{\left[a_{n}+\frac{k_{1}^{k_{1}}}{k^{k}} a_{n}^{-k_{1}}\right] x,} & x \geq m
\end{array}\right\}=\left\{\begin{array}{ll}
V^{(n)}(x, m), & 0 \leq x<m \\
a_{n+1} x, & x \geq m
\end{array}\right\}
$$

by induction hypothesis. Indeed, for every $x \in \mathbb{R}_{+}$, we can calculate $V^{(n)}(x, x)=\left.V^{(n)}(m, m)\right|_{m=x}$ from (13), and the second equality follows from the definition of $a_{n+1}$ in (12). Note that

$$
\limsup _{x \downarrow 0} \frac{g_{m}^{(n+1)}(x)}{\varphi(x)}=\lim _{x \downarrow 0} \frac{m}{x^{-k_{1}}}=0, \quad \limsup _{x \uparrow \infty} \frac{g_{m}^{(n+1)}(x)}{\varphi(x)}=\lim _{x \uparrow \infty} \frac{a_{n+1} x}{x}=a_{n+1}>0 .
$$

Since $a_{n+1}$ is finite, the function $V^{(n+1)}(x, m)$ is finite by Proposition 6(i). Let $W_{m}^{(n+1)}(y)$ be the smallest nonnegative concave majorant of $G_{m}^{(n+1)}(y) \triangleq\left[g_{m}^{(n+1)} / \varphi\right] \circ F^{-1}(y), y \in \mathbb{R}_{+}$. Since $V^{(n)}\left(F^{-1}(y), m\right) / \varphi\left(F^{-1}(y)\right)=W_{m}^{(n)}(y)$ by Proposition 6(ii), we have

$$
G_{m}^{(n+1)}(y)=\left\{\begin{array}{ll}
W_{m}^{(n)}(y), & 0 \leq y<m^{k} \\
a_{n+1} \frac{y^{1 / k}}{y^{-k_{1} / k}}, & y \geq m^{k}
\end{array}\right\}=\left\{\begin{array}{ll}
m y^{k_{1} / k}, & 0 \leq y<y_{m}^{(n)} \\
L_{m}^{(n)}(y), & y_{m}^{(n)} \leq y<m^{k} \\
a_{n+1} y, & y \geq m^{k}
\end{array}\right\}
$$

where the second equality follows from 19 by induction hypothesis, and $L_{m}^{(n)}(y)=a_{n} y+$ $\left(k_{1}^{k_{1}} / k^{k}\right) a_{n}^{-k_{1}} m^{k}$.

Let us now find $W_{m}^{(n+1)}$. It is easy to check that the straight line $y \mapsto L_{m}^{(n)}(y)$ is tangent to the strictly concave and increasing curve $y \mapsto m y^{k_{1} / k}$ at $y=y_{m}^{(n)}<m^{k}$. Moreover, the same line intersects with $y \mapsto a_{n+1} y$ at $y=m^{k}$; see Figure 6(c). Let $y=y_{m}^{(n+1)}$ be the point where the derivative of $y \mapsto m y^{k_{1} / k}$ equals $a_{n+1}$. Since this curve is strictly concave and has infinite right-derivative at $y=0$, the number $y_{m}^{(n+1)}$ exists and is unique and positive. Moreover, $y_{m}^{(n+1)}<y_{m}^{(n)}$, since the derivative of the same concave curve at $y=y_{m}^{(n)}$ equals $a_{n}<a_{n+1}$. Now it is clear that $W_{m}^{(n+1)}(y)$ coincides on $\left[0, y_{m}^{(n+1)}\right]$ with $m y^{k_{1} / k}$ and on $\left[y_{m}^{(n+1)}, \infty\right)$ with the straight line $L_{m}^{(n+1)}(y)$, which has slope $a_{n+1}$ and is tangent to the curve $y \mapsto m y^{k_{1} / k}$ at 

$y=y_{m}^{(n+1)}$. The equations

$$
a_{n+1}=\left.\frac{d}{d y}\left(m y^{k_{1} / k}\right)\right|_{y=y_{m}^{(n+1)}}, \quad L_{m}^{(n+1)}\left(y_{m}^{(n+1)}\right)=\left.m y^{k_{1} / k}\right|_{y=y_{m}^{(n+1)}}
$$

imply that

$$
W_{m}^{(n+1)}(y)= \begin{cases}m y^{k_{1} / k}, & 0 \leq y<y_{m}^{(n+1)}=\left(\frac{k_{1}}{k} \frac{m}{a_{n+1}}\right)^{k}, \\ L_{m}^{(n+1)}(y)=a_{n+1} y+\frac{k_{1}^{k_{1}}}{k^{k}} a_{n+1}^{-k_{1}} m^{k}, & y \geq y_{m}^{(n+1)} .\end{cases}
$$

Finally, if we define $x_{m}^{(n+1)} \triangleq F^{-1}\left(y_{m}^{(n+1)}\right)$, then $V^{(n+1)}(x, m)=\varphi(x) W_{m}^{(n+1)}(F(x))$ gives

$$
V^{(n+1)}(x, m)= \begin{cases}m, & 0 \leq x<x_{m}^{(n+1)}=\frac{k_{1}}{k} \cdot \frac{m}{a_{n+1}}, \\ a_{n+1} x+\frac{k_{1}^{k_{1}}}{k^{k}} m^{k} x^{-k_{1}}, & x \geq x_{m}^{(n+1)}\end{cases}
$$

by Proposition 6(ii). Note that $V_{m}^{(n+1)}$ and $W_{m}^{(n+1)}$ have the same form as in (13) and (19), and the proof of Proposition 3(i) is complete.

Moreover, the second limit in Proposition 6(i) is positive, and the right boundary is again not a limit point of the "stopping region" $\left\{x \in \mathbb{R}_{+}: V^{(n+1)}(x, m)=g_{m}^{(n+1)}(x)\right\}$ unless $m=0$. By Proposition 6(iv), there is no optimal stopping time if and only if $m>0$.

Since $\left(a_{n}\right)_{n \geq 1}$ is increasing, Proposition 3(ii) is obvious. For the proof of (iii), suppose $m>0$ and fix $\varepsilon>0$. Notice that

$$
x_{m}^{(n)}(\varepsilon)=\min \left\{x \geq m ; V^{(n)}(x, m)-g_{m}^{(n)}(x) \leq \varepsilon\right\}=\min \left\{x \geq m ; \frac{k_{1}^{k_{1}}}{k^{k}} a^{-k_{1}} m^{k} x^{-k_{1}} \leq \varepsilon\right\} .
$$

The first exit time $\tau_{m}^{(n)}(\varepsilon)$ of the process $X$ from the open interval $\left(x_{m}^{(n)}, x_{m}^{(n)}(\varepsilon)\right)$ is finite a.s., and the function $x \mapsto V^{(n)}(x, m)$ is $r$-harmonic on the continuation region $\left(x_{m}^{(n)}, \infty\right) \supset$ $\left(x_{m}^{(n)}, x_{m}^{(n)}(\varepsilon)\right)$. Therefore,

$$
\mathbb{E}_{x}\left[e^{-r \tau_{m}^{(n)}(\varepsilon)} g_{m}^{(n)}\left(X_{\tau_{m}^{(n)}(\varepsilon)}\right)\right] \geq \mathbb{E}_{x}\left[e^{-r \tau_{m}^{(n)}(\varepsilon)}\left(V^{(n)}\left(X_{\tau_{m}^{(n)}(\varepsilon)}, m\right)\right)\right]-\varepsilon=V^{(n)}(x, m)-\varepsilon,
$$

and Proposition 3(iii) is proved.

5.2. Proof of Proposition 4. We will prove (i) and (ii) first. By an induction on $n$, we will establish simultaneously the equations (17) and

$$
W_{m}^{(n)}(y)= \begin{cases}m y^{k_{1} / k}, & 0 \leq y<y_{m, 1}^{(n)}<m^{k} \\ L_{m}^{(n)}(y)=m \frac{k_{1}}{k}\left[y_{m, 1}^{(n)}\right]^{-k_{2} / k} y+m \frac{k_{2}}{k}\left[y_{m, 1}^{(n)}\right]^{k_{1} / k}, & y_{m, 1}^{(n)} \leq y<y_{m, 2}^{(n)} \\ a_{n} y^{\left(1+k_{1}\right) / k}, & y \geq y_{m, 2}^{(n)}>m^{k}\end{cases}
$$




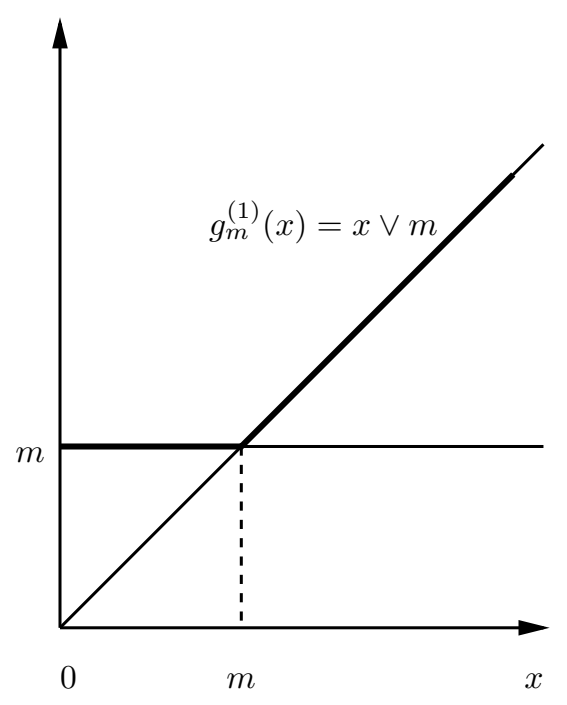

(a)

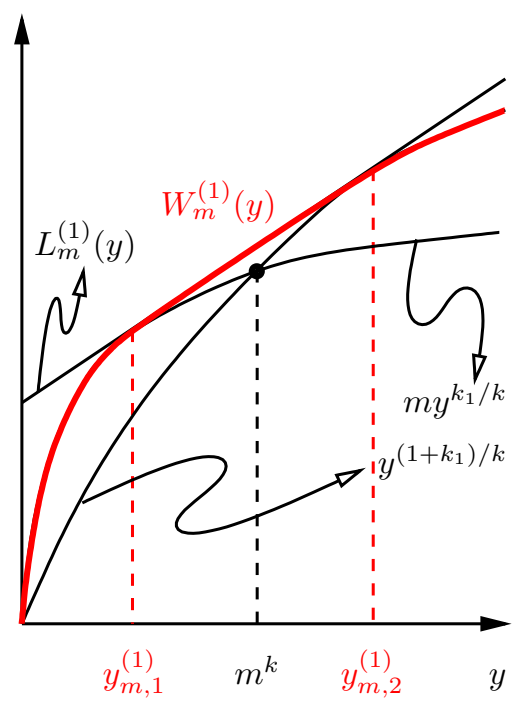

(b)

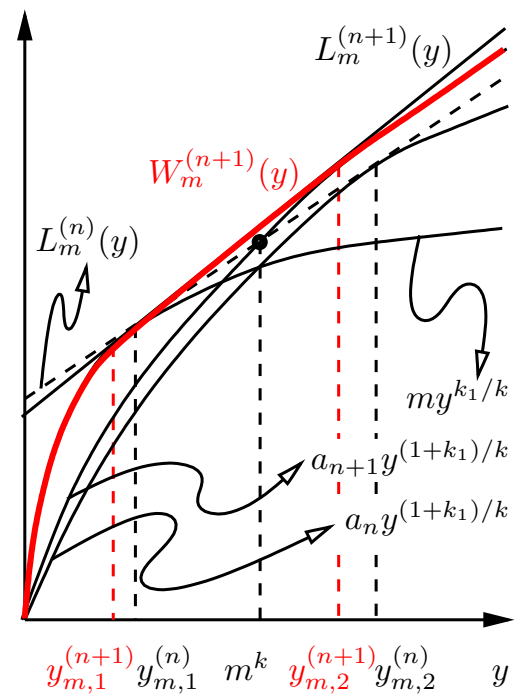

(c)

Figure 7. Illustrations for the proof of Proposition 4. In (b) and (c), $L_{m}^{(n)}(y)$ is the straight line which is tangent to strictly increasing and concave curves $y \mapsto m y^{k_{1} / k}$ and $y \mapsto a_{n} y^{\left(1+k_{1}\right) / k}$ at $y=y_{m, 1}^{(n)}$ and $y=y_{m, 2}^{(n)}$, respectively. In (b), $W_{n}^{(1)}(y)$ is the smallest nonnegative concave majorant of the function $G_{m}^{(1)}(y)$, which is the maximum of the curves $y \mapsto m y^{k_{1} / k}$ and $y \mapsto y^{\left(1+k_{1}\right) / k}$. In (c), $W_{m}^{(n+1)}$ is the same majorant of $G_{m}^{(n+1)}(y)$, which coincides on $\left[0, y_{m, 1}^{(n)}\right]$ with $m y^{k_{1} / k}$, on $\left[y_{m, 1}^{(n)}, m^{k}\right]$ with $L_{m}^{(n)}(y)$, and on $\left[m^{k}, \infty\right)$ with $a_{n+1} y^{\left(1+k_{1}\right) / k}$. The curves $y \mapsto a_{n} y^{\left(1+k_{1}\right) / k}$ and $y \mapsto a_{n+1} y^{\left(1+k_{1}\right) / k}$ intersect at $y=m^{k}$.

where

$$
y_{m, 1}^{(n)}=\left(\frac{k_{1}}{1+k_{1}}\right)^{1+k_{1}}\left(\frac{k_{2}}{k_{2}-1}\right)^{k_{2}-1}\left(\frac{m}{a_{n}}\right)^{k}, \quad y_{m, 2}^{(n)}=\left(\frac{k_{1}}{1+k_{1}}\right)^{k_{1}}\left(\frac{k_{2}}{k_{2}-1}\right)^{k_{2}}\left(\frac{m}{a_{n}}\right)^{k}
$$

are the tangent points of the straight line $y \mapsto L_{m}^{(n)}(y)$ to $y \mapsto G_{m}^{(n)}(y)$ defined in Proposition 6 (ii).

For $n=1$, we have $g_{m}^{(1)}(x)=m \vee x$. Since

$$
\limsup _{x \downarrow 0} \frac{g_{m}^{(1)}(x)}{\varphi(x)}=\limsup _{x \uparrow \infty} \frac{g_{m}^{(1)}(x)}{\psi(x)}=0,
$$

the value function $V^{(1)}(\cdot, m)$ is finite and admits an optimal stopping time by Proposition 6 (i) and (iv). Let us calculate the smallest nonnegative concave majorant $W_{m}^{(1)}(y)$ of the function

$$
G_{m}^{(1)}(y)=\frac{g^{(1)}}{\varphi} \circ F^{-1}(y)= \begin{cases}m y^{k_{1} / k}, & 0 \leq y<m^{k} \\ y^{\left(1+k_{1}\right) / k}, & y \geq m^{k}\end{cases}
$$


The function $y \mapsto G_{m}^{(1)}(y)$ is the maximum of the strictly concave increasing functions $y \mapsto$ $m y^{k_{1} / k}$ and $y \mapsto y^{\left(1+k_{1}\right) / k}$, which intersect at $y=m^{k}$; see Figure 7 (b). The valley centered at $y=m^{k}$ can be bridged by a straight line $L_{m}^{(1)}$ which is tangent to $y \mapsto m y^{k_{1} / k}$ and $y \mapsto y^{\left(1+k_{1}\right) / k}$ at $y=y_{m, 1}^{(1)}$ and $y=y_{m, 2}^{(1)}$, respectively, and majorizes $G_{m}^{(1)}$ everywhere. The points $y_{m, 1}^{(1)}$ and $y_{m, 2}^{(1)}$ are unique solutions $u<m^{k}<v$ of the system of equations

$$
\left.\frac{d}{d y}\left(m y^{k_{1} / k}\right)\right|_{y=u}=\frac{v^{\left(1+k_{1}\right) / k}-m u^{k_{1} / k}}{v-u}=\left.\frac{d}{d y}\left(y^{\left(1+k_{1}\right) / k}\right)\right|_{y=v} .
$$

The straight-forward calculations give

$$
y_{m, 1}^{(1)}=\left(\frac{k_{1}}{1+k_{1}}\right)^{1+k_{1}}\left(\frac{k_{2}}{k_{2}-1}\right)^{k_{2}-1} m^{k}<m^{k}<y_{m, 2}^{(1)}=\left(\frac{k_{1}}{1+k_{1}}\right)^{k_{1}}\left(\frac{k_{2}}{k_{2}-1}\right)^{k_{2}} m^{k} .
$$

Moreover, the equation of the straight line $L_{m}^{(1)}(y)$ becomes

$$
G_{m}^{(1)}\left(y_{m, 1}^{(1)}\right)+\left.\left(y-y_{m, 1}^{(1)}\right) \cdot \frac{d}{d y} G_{m}^{(1)}(y)\right|_{y=y_{m, 1}^{(1)}}=m \frac{k_{1}}{k}\left[y_{m, 1}^{(1)}\right]^{-k_{2} / k} y+m \frac{k_{2}}{k}\left[y_{m, 1}^{(1)}\right]^{k_{1} / k} .
$$

Therefore, we have

$$
W_{m}^{(1)}(y)= \begin{cases}m y^{k_{1} / k}, & 0 \leq y<y_{m, 1}^{(1)}<m^{k} \\ L_{m}^{(1)}(y), & y_{m, 1}^{(1)} \leq y<y_{m, 2}^{(1)} \\ y^{\left(1+k_{1}\right) / k}, & y \geq y_{m, 2}^{(1)}>m^{k} .\end{cases}
$$

If we define $x_{m, j}^{(1)}=F^{(-1)}\left(y_{m, j}^{(1)}\right)=\left(y_{m, j}^{(1)}\right)^{1 / k}$ for $j=1,2$, then Proposition 6(ii) implies that $V^{(1)}(x, m)=\varphi(x) W_{m}^{(1)}(F(x))$; i.e., with $\ell_{m}^{(1)}(x)=x^{-k_{1}} L_{m}^{(1)}\left(x^{k}\right)$ we have

$$
V^{(1)}(x, m)= \begin{cases}m, & 0 \leq x<x_{m, 1}^{(1)}<m^{k}, \\ \ell_{m}^{(1)}(x)=\frac{m}{k}\left[k_{1}\left(\frac{x}{x_{m, 1}^{(1)}}\right)^{k_{2}}+k_{2}\left(\frac{x}{x_{m, 1}^{(1)}}\right)^{-k_{1}}\right], & x_{m, 1}^{(1)} \leq x<x_{m, 2}^{(1)}, \\ x, & x \geq x_{m, 2}^{(1)} .\end{cases}
$$

This proves part (i) for $n=1$. Suppose that $V^{(n)}(x, m)$ and $W_{m}^{(n)}(y)$ are given by 15 and (20), respectively, for some $n \geq 1$. Let us show that they also hold for $n+1$. It is easy to check that

$$
\begin{aligned}
V^{(n)}(m, m)=\ell_{m}^{(n)}(m)=m\{ & \frac{k_{1}}{k}\left[\left(\frac{k_{1}}{1+k_{1}}\right)^{1+k_{1}}\left(\frac{k_{2}}{k_{2}-1}\right)^{k_{2}-1}\right]^{-k_{2} / k}\left(a_{n}\right)^{k_{2}} \\
+ & \left.\frac{k_{2}}{k}\left[\left(\frac{k_{1}}{1+k_{1}}\right)^{1+k_{1}}\left(\frac{k_{2}}{k_{2}-1}\right)^{k_{2}-1}\right]^{k_{1} / k}\left(a_{n}\right)^{-k_{1}}\right\}=a_{n+1} m
\end{aligned}
$$


by the definition of $a_{n+1}$ in (14). Therefore,

$$
g_{m}^{(n+1)}(x)=V^{(n)}(x, m \vee x)= \begin{cases}V^{(n)}(x, m), & 0 \leq x<m, \\ a_{n+1} x, & x \geq m,\end{cases}
$$

and since $V^{(n)}\left(F^{-1}(y), m\right) / \varphi\left(F^{-1}(y)\right)=W_{m}^{(n)}(y)$ by Proposition 6(ii), we have

$$
G_{m}^{(n+1)}(y)=\left\{\begin{array}{ll}
W_{m}^{(n)}(y), & 0 \leq y<m^{k} \\
a_{n+1} y^{\left(1+k_{1}\right) / k}, & y \geq m^{k}
\end{array}\right\}=\left\{\begin{array}{ll}
m y^{k_{1} / k}, & 0 \leq y<y_{m, 1}^{(n)} \\
L_{m}^{(n)}(y), & y_{m, 1}^{(n)} \leq y<m^{k} \\
a_{n+1} y^{\left(1+k_{1}\right) / k}, & y \geq m^{k}
\end{array}\right\}
$$

by the induction hypothesis. The function $y \mapsto G_{m}^{(n+1)}$ is the maximum of the concave and increasing curves $y \mapsto W_{m}^{(n)}(y)$ and $y \mapsto a_{n+1} y^{\left(1+k_{1}\right) / k}$, which meet at $y=m^{k}$; see Figure 7 (c). The valley centered at $y=m^{k}$ in the graph of $y \mapsto G_{m}^{(n+1)}$ can be bridged by a straight line $L_{m}^{(n+1)}(y)$, which majorizes $G_{m}^{(n+1)}$ everywhere and is tangent to the curves $y \mapsto W_{m}^{(n)}(y)$ and $y \mapsto a_{n+1} y^{\left(1+k_{1}\right) / k}$ at some points $y=y_{m, 1}^{(n+1)}$ and $y=y_{m, 2}^{(n+1)}$, respectively. Since $y \mapsto a_{n+1} y^{\left(1+k_{1}\right) / k}$ is above the line $L_{m}^{(n)}(y)$ on $y \in\left[m^{k}, \infty\right)$, the points $y_{m, 1}^{(n+1)}$ and $y_{m, 2}^{(n+1)}$ are the unique solutions $u<y_{m, 1}^{(n)}<m^{k}<v$ of

$$
\left.\frac{d}{d y}\left(m y^{k_{1} / k}\right)\right|_{y=u}=\frac{a_{n+1} v^{\left(1+k_{1}\right) / k}-m u^{k_{1} / k}}{v-u}=\left.\frac{d}{d y}\left(a_{n+1} y^{\left(1+y_{1}\right) / k}\right)\right|_{y=v} .
$$

After straight-forward algebra, we obtain

$$
\begin{aligned}
y_{m, 1}^{(n+1)} & =\left(\frac{k_{1}}{1+k_{1}}\right)^{1+k_{1}}\left(\frac{k_{2}}{k_{2}-1}\right)^{k_{2}-1}\left(\frac{m}{a_{n+1}}\right)^{k}<y_{m, 1}^{(n)}<m^{k}, \\
m^{k}<y_{m, 2}^{(n+1)} & =\left(\frac{k_{1}}{1+k_{1}}\right)^{k_{1}}\left(\frac{k_{2}}{k_{2}-1}\right)^{k_{2}}\left(\frac{m}{a_{n+1}}\right)^{k}<y_{m, 2}^{(n)} .
\end{aligned}
$$

Last inequality follows from that $a_{n+1}>a_{n}$. The equation of the line $L_{m}^{(n+1)}(y)$ becomes $W_{m}^{(n)}\left(y_{m, 1}^{(n+1)}\right)+\left.\left(y-y_{m, 1}^{(n+1)}\right) \cdot \frac{d}{d y} W_{m}^{(n)}(y)\right|_{y=y_{m, 1}^{(n+1)}}=m \frac{k_{1}}{k}\left[y_{m, 1}^{(n+1)}\right]^{-k_{2} / k} y+m \frac{k_{2}}{k}\left[y_{m, 1}^{(n+1)}\right]^{k_{1} / k}$, and the smallest nonnegative concave majorant of $G_{m}^{(n+1)}$ is given by

$$
W_{m}^{(n+1)}(y)= \begin{cases}m y^{k_{1} / k}, & 0 \leq y<y_{m, 1}^{(n+1)}<y_{m, 1}^{(n)}<m^{k} \\ L_{m}^{(n+1)}(y), & y_{m, 1}^{(n+1)} \leq y<y_{m, 2}^{(n+1)}<y_{m, 2}^{(n)} \\ y^{\left(1+k_{1}\right) / k}, & y \geq y_{m, 2}^{(n+1)}>m^{k}\end{cases}
$$

which is the same as 20 with $n+1$ instead of $n$. Finally, let us define $x_{m, j}^{(n+1)}=F^{-1}\left(y_{m, j}^{(n+1)}\right)=$ $\left(y_{m, j}^{(n+1)}\right)^{1 / k}$ for $j=1,2$. Then $x_{m, 1}^{(n)}<x_{m, 1}^{(n+1)}<m^{k}<x_{m, 2}^{(n+1)}<x_{m, 2}^{(n)}$ proves (ii), and by 
Proposition 6(ii) we have that $V^{(n+1)}(x, m)=\varphi(x) W_{m}^{(n+1)}(F(x))$ equals

$$
V^{(n+1)}(x, m)= \begin{cases}m, & 0 \leq x<x_{m, 1}^{(n+1)}, \\ \ell_{m}^{(n+1)}(x)=\frac{m}{k}\left[k_{1}\left(\frac{x}{x_{m, 1}^{(n+1)}}\right)^{k_{2}}+k_{2}\left(\frac{x}{x_{m, 1}^{(n+1)}}\right)^{-k_{1}}\right], & x_{m, 1}^{(n+1)} \leq x<x_{m, 1}^{(n+1)}, \\ a_{n+1} x, & x \geq x_{m, 2}^{(n+1)}\end{cases}
$$

in terms of $\ell_{m}^{(n+1)}(x) \triangleq x^{-k_{1}} L_{m}^{(n+1)}\left(x^{k}\right)$. This completes the proof of Proposition 4 (i) and (ii). Finally, (iii) follows from Proposition 6 (iii).

5.3. Proof of Proposition 5. We need to prove only the first equality; the rest follows immediately from it. The sequence $\left(a_{n}\right)_{n \geq 1}$ in $(14)$ is increasing. Therefore, the limit $a \triangleq$ $\lim _{n \rightarrow \infty} a_{n} \geq 1$ exists and satisfies the equation

$$
\begin{aligned}
x=\frac{k_{1}}{k}\left[\left(\frac{k_{1}}{1+k_{1}}\right)^{1+k_{1}}\left(\frac{k_{2}}{k_{2}-1}\right)^{k_{2}-1}\right]^{-k_{2} / k} & x^{k_{2}} \\
& +\frac{k_{2}}{k}\left[\left(\frac{k_{1}}{1+k_{1}}\right)^{1+k_{1}}\left(\frac{k_{2}}{k_{2}-1}\right)^{k_{2}-1}\right]^{k_{1} / k} x^{-k_{1}},
\end{aligned}
$$

obtained by passing to limit as $n \rightarrow \infty$ in (14). In order to find $a$, we will guess its value, verify that it satisfies the above equation, and that the equation has exactly one solution.

From Proposition 4 (i) and (ii) we know that

$$
x_{m, 2}^{(n)} \triangleq\left(\frac{k_{1}}{1+k_{1}}\right)^{k_{1} / k}\left(\frac{k_{2}}{k_{2}-1}\right)^{k_{2} / k} \frac{m}{a_{n}}>m, \quad n \geq 1 .
$$

Since $a_{n} \nearrow a$, this inequality implies that $a$ is finite. Recall that $x_{m, 2}^{(n)}$ gives the upper exercise threshold when there are $n$ exercise rights. Intuitively, as the number of exercise rights increases, the optimal waiting time before marking a new record of the process $X$ should get shorter. In the limit, this waiting time should reduce to zero if the process starts at $m$. Therefore, we expect $\lim _{n \rightarrow \infty} x_{m, 2}^{(n)}=m$, which implies that $a$ equals

$$
\widehat{a}=\left(\frac{k_{1}}{1+k_{1}}\right)^{k_{1} / k}\left(\frac{k_{2}}{k_{2}-1}\right)^{k_{2} / k}
$$

It is easy to verify that $\widehat{a}$ satisfies 21 .

To show that $a=\widehat{a}$, we shall prove that $\widehat{a}$ is the unique solution of (21). Note that every $x$ satisfying (21) must be nonzero. If we divide (21) by $\varphi(x)=x^{-k_{1}}$ and replace in the 
resulting equation every $x$ with $F^{-1}(x)=x^{1 / k}$, we obtain

$$
\begin{array}{r}
x^{\left(1+k_{1}\right) / k}=\frac{k_{1}}{k}\left[\left(\frac{k_{1}}{1+k_{1}}\right)^{1+k_{1}}\left(\frac{k_{2}}{k_{2}-1}\right)^{k_{2}-1}\right]^{-k_{2} / k} x \\
+\frac{k_{2}}{k}\left[\left(\frac{k_{1}}{1+k_{1}}\right)^{1+k_{1}}\left(\frac{k_{2}}{k_{2}-1}\right)^{k_{2}-1}\right]^{k_{1} / k} .
\end{array}
$$

Note that $x$ solves $(21)$ if and only if $F(x)=x^{k}$ solves $(22)$. Therefore, it is enough to show that $F(\widehat{a})=\widehat{a}^{k}$ is the unique solution of 22 . However, its easy to check that the straight line on the right is tangent to the strictly concave curve on the left exactly at $x=\widehat{a}$; because of the properties of strictly concave functions, they cannot meet at anywhere else.

\section{Value of Russian option as limit of MUltiple-Stopping PROBlems}

In all the examples studied above, in the limit as $n$ goes to infinity, the multiple-stopping value function $V^{(n)}(x, 0)$ converges to the value of the Russian option $V(x)$. This fact can be shown to hold for quite general processes $X$ and provides an alternative method of obtaining $V(x)$. Let $V^{(\infty)}(x, m)=\lim _{n \rightarrow \infty} V^{(n)}(x, m)$. Then $V(x)=V^{(\infty)}(x, 0)$ is a fixed point of the iteration performed in (4). Indeed, $V^{(\infty)}(x, m)$ is the smallest concave majorant of $g_{m}^{(\infty)}(x) \triangleq V^{(\infty)}(x, h(x) \vee m)$ for any $x, m \in \mathcal{I}$. This observation leads to an ordinary differential equation (ODE) that must be satisfied by $V^{(\infty)}(x, m)$ from the slope-matching conditions.

To illustrate this idea heuristically, consider the problem solved in Proposition 1. Recall

$$
V^{(\infty)}(x, m)= \begin{cases}m-\log (m) x, & x \leq m, \\ x-x \log (x), & x>m .\end{cases}
$$

From Figure 5 it is easy to see that $V^{(\infty)}(x, m)$ must consist of a linear segment between $\left[0, x_{m}^{(\infty)}\right)$ and the curve $V(x) \equiv V^{(\infty)}(x, 0)$ on $\left[x_{m}^{(\infty)}, 1\right]$-observe that for $x \geq x_{m}^{(n)}$, the function $V^{(n)}(x, m)$ is independent of $m$. Moreover, since with infinite number of exercise rights we exercise whenever a new record is achieved, we must have $x_{m}^{(\infty)}=m$. From the graph we also see that the two pieces should be smoothly connected at $x_{m}^{(\infty)}$. The latter slope-matching condition at $x_{m}^{(\infty)}=m$ together with $V^{(\infty)}(m, m)=V(m)$ now implies that

$$
[V(m)-m] / m=V^{\prime}(m) .
$$

The left-hand side above is equal to the slope of $V^{(\infty)}(x, m)$ between the points $(0, m)$ and $\left(x_{m}^{(\infty)}, V\left(x_{m}^{(\infty)}\right)\right)$, which is also the left-derivative of $V^{(\infty)}(x, m)$ at $x_{m}^{(\infty)}$. The right-hand side of (23) is the derivative of $V(x)$ at $x=m$; i.e., the right-derivative of $V^{(\infty)}(x, m)$ at $x=m$. 
Moreover, we have the boundary condition $V(1)=1$. Solving the first-order ODE in (23) we immediately get $V(x)=x(1-\log (x))$, as already shown in (8).

Applying the same method to the problem of Proposition 2, we obtain $x_{m}^{(\infty)}=K-m$ and $V^{(\infty)}(x, m)$ is $V(x)$ on $\left[0, x_{m}^{(\infty)}\right)$ and is affine on $\left[x_{m}^{(\infty)}, 1\right]$. The function $V(x)$ itself is linear on $[K, 1]$, since on the latter interval the reward is identically zero. The slope-matching at $x_{m}^{(\infty)}$ reduces to the first-order ODE

$$
\frac{V(K-m)-m}{(K-m)-1}=V^{\prime}(K-m),
$$

with the boundary condition $V(0)=K$. Solving (24) we recover the function in (18).

In general, let $H(y) \triangleq 1 / \varphi\left(F^{-1}(y)\right)$ as defined in Section 3. We work in the transformed $y$-space determined by Proposition 6. In the canonical situation the continuation region is connected and determined by upper and lower boundaries $y_{m, 1}^{(n)}<F(m)<y_{m, 2}^{(n)}$. As in Proposition 5. $y_{m, 1}^{(n)} \searrow \hat{y}$ decreases to some limit $\hat{y}$, while $y_{m, 2}^{(n)} \searrow F(m)$, since with unlimited number of exercises it is optimal to stop as soon as a new record is set; i.e., as soon as $F(X)$ enters $(F(m), \infty)$. Accordingly, we must have

$$
W_{m}^{(\infty)}(y)= \begin{cases}m H(y), & y \leq \hat{y}, \\ l_{m}(y) \triangleq f(m) y+m H(\hat{y})-f(m) \hat{y}, & \hat{y}<y<F(m), \\ L(y) \triangleq l_{F^{-1}(y)}(y), & y \geq F(m)\end{cases}
$$

for some function $f(\cdot)$. Namely, for $x$ very small, $V^{(\infty)}(x, m)=m$, which implies $W_{m}^{(\infty)}(y)=$ $m H(y)$. In the continuation region $\left(y_{m, 1}^{(\infty)}, y_{m, 2}^{(\infty)}\right)$, the function $W_{m}^{(\infty)}(y)$ is affine, while for large $x$ it is optimal to stop immediately and the transformed reward is $\left.W_{m}^{(\infty)}(y)\right|_{m=F^{-1}(y)}$.

Since $W_{m}^{(\infty)}$ is a limit of $W_{m}^{(n)}$, it must be invariant with respect to taking concave majorants. As a result, $W_{m}^{(\infty)}(y)$ is itself concave and its slope at the upper threshold $F(m)$ must equal its slope at the lower threshold $\hat{y}$, as well as the slope of the affine segment. It follows that the only unknowns above are (i) the function $f(\cdot)$, which gives the slope of the linear portion of $W_{m}^{(\infty)}(y)$ as a function of $m$, and (ii) the threshold $\hat{y}(m)$, which indicates the beginning of this segment. The slope-matching conditions require

$$
\left.\frac{d}{d y} L(y)\right|_{y=F(m)}=f(m)=\left.m \frac{d}{d y} H(y)\right|_{y=\hat{y}} .
$$

The second equality can be used to find the lower threshold $\hat{y}(m)$, while the other equality can be reduced to

$$
f^{\prime}(m)(\hat{y}-F(m))=H(\hat{y})
$$


Unfortunately, there is no natural boundary condition for selecting a particular solution of (27), so to obtain a full characterization of $W_{m}^{(\infty)}$ each problem must be considered on its own.

Remark 4. For geometric Brownian motion on $\mathcal{I}=\mathbb{R}_{+}$with $h(x)=x$, this fixed point method implies that $f(m)$ of $(25)$ solves

$$
f^{\prime}(m)=\frac{\hat{y}^{k_{1} / k}}{\hat{y}-m^{k}} \quad \text { where } \quad \hat{y}=\left(\frac{f(m)}{m} \frac{k}{k_{1}}\right)^{-k / k_{2}} .
$$

It can verified that this ODE is indeed satisfied by $f(m)=m \frac{k_{1}}{k}(\hat{y})^{-k_{2} / k}$ from (20) with $\hat{y}=\left(\frac{k_{1}}{1+k_{1}}\right)^{1+k_{1}}\left(\frac{k_{2}}{k_{2}-1}\right)^{k_{2}-1}\left(\frac{m}{a}\right)^{k}$ and $a$ of Proposition 5 .

Finally, let us turn our attention to the unsolved problem of a Russian option for arithmetic Brownian motion with drift. Suppose that $r>0$ and let

$$
X_{t}=x+\mu t+\sigma W_{t}, \quad t \geq 0, x \in \mathcal{I}=\mathbb{R},
$$

be a Brownian motion with drift $\mu$ and volatility $\sigma$. Denote by $k_{1,2}$ the roots of the quadratic equation $\left(\sigma^{2} / 2\right) k^{2}+\mu k-r=0$; namely,

$$
-k_{1}=-\frac{\mu}{\sigma^{2}}-\sqrt{\frac{\mu^{2}}{\sigma^{4}}+\frac{2 r}{\sigma^{2}}}<0<k_{2}=-\frac{\mu}{\sigma^{2}}+\sqrt{\frac{\mu^{2}}{\sigma^{4}}+\frac{2 r}{\sigma^{2}}} ; \quad \text { and } \quad k=k_{2}+k_{1} .
$$

It is easy to check that at each step $W_{m}^{(n)}(y)$ has three pieces: an initial one of the form $m y^{k_{1} / k}$ for $y \leq y_{m, 1}^{(n)}$, then an affine segment for $y_{m, 1}^{(n)}<y<y_{m, 2}^{(n)}$, followed by a concave piece on $y \geq y_{m, 2}^{(n)}$. Unfortunately, explicit computations are not possible. Already the equation defining $V^{(2)}$ does not have closed-form formulas. However, the preceding method does lead to the following conjecture:

Conjecture. If $X$ is an arithmetic Brownian motion, $r>0$ and $h(x)=x$, then the limiting value as $n \rightarrow \infty$ of the functions in (3) is given by

$$
V^{(\infty)}(x, m)= \begin{cases}m, & x \leq \frac{1}{k} \log \left(y_{m, 1}\right), \\ l_{m}(x) \triangleq f(m) e^{k_{2} x}+\left(m y_{m, 1}^{k_{1} / k}-f(m) y_{m, 1}\right) e^{-k_{1} x}, & \frac{1}{k} \log \left(y_{m, 1}\right)<x<m, \\ L(x) \triangleq l_{x}(x), & x \geq m .\end{cases}
$$

The function $f(\cdot)$ satisfies

$$
f^{\prime}(m)=\frac{\left(\frac{f(m)}{m} \frac{k}{k_{1}}\right)^{-k_{1} / k_{2}}}{\left(\frac{f(m)}{m} \frac{k}{k_{1}}\right)^{-k / k_{2}}-e^{k m}} \quad \text { with } \quad y_{m, 1}=\left(\frac{f(m)}{m} \frac{k}{k_{1}}\right)^{-k / k_{2}} \text { and } \quad \lim _{m \rightarrow \infty} f(m)=0 .
$$


The boundary condition of (30) is based on the observation that any solution of the corresponding ODE has a horizontal asymptote for large $m$. On the other hand, as $m \rightarrow \infty$, $y_{m, 1} \rightarrow e^{k m}$ because the exponential cost of waiting dominates the potential linear gain. Consequently, the continuation region shrinks and the slope of the affine segment goes to zero. Numerical results indicate that the desired solution might also be a separatrix; namely, the smallest non-exploding solution of (30). If our conjecture is true, then the value of the Russian option in this case is $V(x)=V^{(\infty)}(x, 0)=L(x)$ for any $x \in \mathbb{R}_{+}$.

\section{ACKNOWLEDGMent}

This work was initiated while both authors were attending the Symposium on Optimal Stopping with Applications in Manchester, United Kingdom between 22-27 January 2006. They are very grateful to the organizers for this stimulating event.

\section{REFERENCES}

[1] René Carmona and Savas Dayanik, Optimal multiple-stopping of linear diffusions and swing options, Preprint. (http://www.princeton.edu/ sdayanik/papers/swing.pdf), 2003.

[2] René Carmona and Nizar Touzi, Optimal multiple stopping and valuation of swing options, to appear in Mathematical Finance, 2003.

[3] Savas Dayanik and Ioannis Karatzas, On the optimal stopping problem for one-dimensional diffusions, Stochastic Process. Appl. 107 (2003), no. 2, 173-212. MR MR1999788 (2004d:60104)

[4] J. Darrell Duffie and J. Michael Harrison, Arbitrage pricing of Russian options and perpetual lookback options, Ann. Appl. Probab. 3 (1993), no. 3, 641-651. MR MR1233618 (94i:90028)

[5] E. B. Dynkin, Optimal choice of the stopping moment of a Markov process, Dokl. Akad. Nauk SSSR 150 (1963), 238-240. MR MR0154329 (27 \#4278)

[6] Evgenii B. Dynkin and Aleksandr A. Yushkevich, Markov processes: Theorems and problems, Translated from the Russian by James S. Wood, Plenum Press, New York, 1969. MR MR0242252 (39 \#3585a)

[7] P. R. Freeman, The secretary problem and its extensions: a review, Internat. Statist. Rev. 51 (1983), no. 2, 189-206. MR MR715534 (84k:62115)

[8] Stephen M. Samuels, Secretary problems, Handbook of sequential analysis, Statist. Textbooks Monogr., vol. 118, Dekker, New York, 1991, pp. 381-405. MR MR1174312

[9] L. A. Shepp and A. N. Shiryaev, A new look at the "Russian option", Teor. Veroyatnost. i Primenen. 39 (1994), no. 1, 130-149. MR MR1348192 (97e:90007)

[10] Larry Shepp and A. N. Shiryaev, The Russian option: reduced regret, Ann. Appl. Probab. 3 (1993), no. 3, 631-640. MR MR1233617 (94i:90027)

(S. Dayanik) Department of Operations Research and Financial Engineering, And Bendheim Center for Finance, Princeton University, Princeton, NJ 08544

E-mail address: sdayanik@princeton.edu

(M. Ludkovski) Department of Mathematics, University of Michigan, Ann Arbor, Mi 48109 E-mail address: mludkov@umich.edu 